\title{
Hydration lubrication
}

\author{
Jacob KLEIN* \\ Department of Materials \& Interfaces, Weizmann Institute of Science, Rehovot, 76100, Israel \\ Received: 28 November 2012 / Revised: 21 January 2013/ Accepted: 25 January 2013 \\ (C) The author(s) 2013. This article is published with open access at Springerlink.com
}

\begin{abstract}
The hydration lubrication paradigm, whereby hydration layers are both strongly held by the charges they surround, and so can support large pressures without being squeezed out, and at the same time remain very rapidly relaxing and so have a fluid response to shear, provides a framework for understanding, controlling, and designing very efficient boundary lubrication systems in aqueous and biological media. This review discusses the properties of confined water, which—unlike organic solvents—retains its fluidity down to molecularly thin films. It then describes lubrication by hydrated ions trapped between charged surfaces, and by other hydrated boundary species including charged and zwitterionic polymer brushes, surfactant monolayers, liposomes, and biological macromolecules implicated in synovial joint lubrication. Finally, challenges and prospects for future development of this new boundary lubrication approach are considered.
\end{abstract}

Keywords: hydration lubrication; biolubrication; boundary lubrication; liposomes; polymer brushes; hydration repulsion; hydration layers

\section{Introduction}

Attempts to reduce friction between sliding surfaces have been documented since antiquity [1,2], as in the ancient Egyptian wall paintings at Saqqara or in references in the Hebrew Bible [2]. Modern insight into the nature of friction has emerged through understanding the dissipative processes that take place between rubbing surfaces [3, 4], an approach particularly championed by the late David Tabor [5]. The main modes of lubrication are often considered in terms of hydrodynamic, elastohydrodynamic and boundary processes (or a combination of these), as well as processes such as plastic deformation of the substrates and those that result in wear [6-9]. Boundary lubrication [10,11] concerns molecularly thin films of materials (the lubricant) that are attached to the mutually-sliding substrates, so that when the surfaces slide past each other the slip occurs at the interface between these boundary layers.

\footnotetext{
* Corresponding author: Jacob KLEIN.
}

E-mail: Jacob.klein@weizmann.ac.il
The essential energy dissipating process is the one where irreversible processes occur, such as breaking of bonds-e.g., van der Waals bonds-between atoms and molecules in the slip plane as they are forced in and out of contact during the sliding (the ideas underlying this mechanism were first examined by Prandtl [12] and by Tomlinson [13]) These irreversible processes can be described also in terms of adhesion hysteresis, which is the difference between energy cost to separate two surfaces and the energy gain when they are brought together again. Indeed it has been found that in many cases the friction varies with the adhesion hysteresis rather than with the adhesion [14-16]. A classic approach to boundary lubrication utilizes amphiphilic surfactant molecules whose polar head-groups attach to the solid substrate $[7,10]$, forming monolayers with the alkyl tails exposed, leading to relatively low sliding friction and greatly reduced wear of the underlying substrates $[7,17,18]$. In hydrodynamic lubrication processes, the energy dissipation during sliding is a viscous process (generating heat) as the film between the sliding interfaces is sheared [9]. 
Over the past decades the use of polymers and other soft materials adsorbed or grafted as boundary lubricants on surfaces has been examined, particularly when considering friction in liquid environments [19-25]. The way in which the chain-like nature of polymeric layers affects frictional forces, in particular the configurational entropy of chains on the one hand [20, 26, 27], and their topological entanglements on the other [28, 29], is reasonably well understood. The issue of bridging of the gap between surfaces bearing adsorbed polymers is central to understanding why frictional dissipation with adsorbed chains is so much greater than with non-adsorbing (grafted) chains [30]. Friction in a water environment is of special interest. This is because water is the natural medium in the context of biological lubrication processes and of biomedical devices [31-35]. This is therefore an area of enormous applicability and potential for future development, and may have implications also for tissue engineering in regenerative medicine [36], where the interfacial properties of scaffolding materials have not been extensively explored.

Water has many unique properties, but one of them-of particular relevance to friction and lubrication processes-was discovered only relatively recently. This is the persistent fluidity of water in confined thin films [37, 38]. Non-associating liquids, including organic solvents and oils, are known to become solid-like, with a viscosity which diverges, when confined between two surfaces as they slide past each other across films that are just a few monolayers thick $[39,40]$. In contrast, water retains a bulk-like fluidity even when confined between solid surfaces to films that are down to 1 monolayer in thickness [37]. This has crucial consequences for flow and frictional effects in living systems.

The "ultimate" thin water films may be viewed as the hydration layers that form about charges in aqueous media as a result of the large dipole of the water molecule [41]; such hydration layers turn out to have truly remarkable properties in the context of lubrication. In the past decade or so, several studies have uncovered a mode of lubrication in aqueous systems, termed hydration lubrication [37, 42-46].
The origin of this is in the hydration layers that form about charges. A sheath or shell of such molecules surrounding a charge [47] may be very strongly attached [48] and at the same time very rapidly relaxing [41]. Thus such a hydrated charge between sliding surfaces can sustain a large normal load because of the reluctance of the hydration water to be squeezed out, but under shear this hydration layer responds in a fluid manner because of its rapid relaxation. This combination of sustaining a large normal load together with a fluid response to shear [45], has been termed the hydration lubrication mechanism. It can lead to a striking reduction of friction between surfaces which expose or slide across such hydrated layers, an effect which has now been observed in many of different systems. This review will discuss our present knowledge and understanding of this effect, which provides a new paradigm for boundary lubrication processes: One which relies on the lubricating elements formed by hydrated charges exposed at sliding interfaces.

In what follows we first consider the issue and origins of the persistent fluidity of water in thin films, in strong contrast to organic liquids and oils. We then discuss the nature of hydration layers and the basic experiments that revealed the hydration lubrication effect. Following this, we describe a number of systems where this effect has been observed and studied: lubrication by charged and zwitterionic polymer brushes; boundary lubrication by classical surfactants under water; and the remarkable effects observed when liposomes-vesicles consisting of phospholipids, the building blocks of living cell membranes - are attached as boundary layers at surfaces. We follow this by discussing the relation of hydration lubrication to biological friction, in particular for the major load bearing joints such as hips or knees. We conclude this review by emphasizing the challenges and opportunities that lie in the understanding and utilization of this new lubrication paradigm.

\section{Fluidity of water in thin confined films}

When two solid surfaces approach each other across a fluid, they may experience both long-ranged and 
short-ranged forces $[47,49,50]$. The former may be due to van der Waals forces that act between all surfaces, or-in the case of like-charged surfaces in aqueous media-they often arise from so-called double layer electrostatic repulsions that are due to the osmotic pressure of trapped counterions (and so are essentially entropic in character). Short-ranged or steric forces may arise from the interactions of surface-attached molecules of the confined liquids, sometimes called solvation or structural forces (or hydration forces, see below, when in aqueous media). A typical example of the normal force $\left(F_{\mathrm{n}}(D)\right)$ vs. surface separation $(D)$ profile between two curved, charged mica surfaces across water, measured using a surface force balance (SFB), is shown in Fig. 1.

The force is normalized by the radius of curvature $R$ of the surfaces as $F(D) / R$, which, as long as $R>>D$, is related via the Derjaguin approximation $[47,49]$ to the surface energy per unit area $E(D)$ of flat parallel surfaces obeying the same force-distance law, as $F(D) / R=2 \pi E(D)$. Since $R \approx 1 \mathrm{~cm}$ while $D \approx 100 \mathrm{~nm}$, this approximation is valid. Figure 1 shows the long-ranged repulsion arising from the electrostatic double-layer on each surface, commencing at some

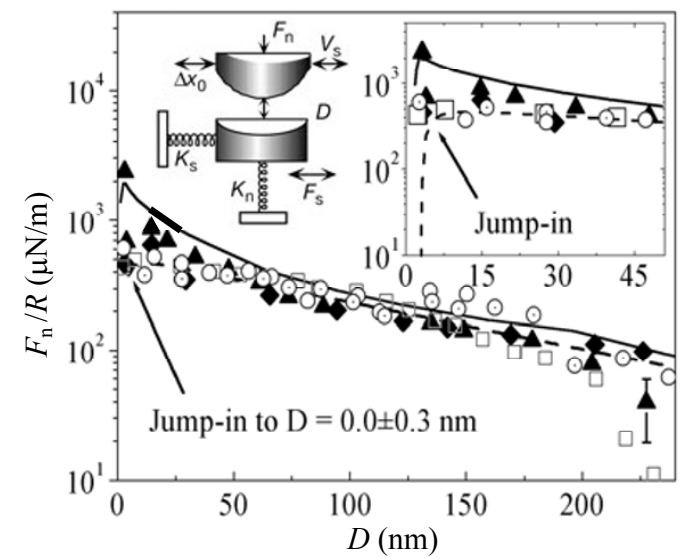

Fig. 1 Force $\left(F_{\mathrm{n}}\right)$ vs. surface separation $D$ profile, measured in the $\mathrm{SFB}$, normalized as $F_{\mathrm{n}} / R$ where $R(\approx 1 \mathrm{~cm})$ is the surface radius of curvature (in the Derjaguin approximation $F_{\mathrm{n}} / R$ yields the surface energy/unit area), between bare mica surfaces across water. The main figure shows the long-ranged electrostatic double layer repulsion-Eq. 1 in text-while the inset shows the jump-in to contact under van der Waals forces from $D \approx 4 \mathrm{~nm}$. The inset cartoon illustrates the SFB configuration, where the upper surface can be moved normally to change $D$ or laterally (via a sectored piezoelectric tube), and normal and shear forces are measured via the bending of the two orthogonal springs $K_{\mathrm{n}}$ and $K_{\mathrm{s}}$ respectively. (adapted from Ref. [37]) hundreds of nanometers; when the surfaces approach to a few nanometer separation, van der Waals attractions exceed this repulsion, and at $D \approx 4 \mathrm{~nm}$ the surfaces jump into adhesive van der Waals contact $(D=0)$ as indicated in the inset. The overall force profile in such a system is well described by the Derjaguin-Landau-Vervey-Overbeek (DLVO) expression [47]:

$F_{\mathrm{n}} / R=128 \pi c \mathrm{k}_{\mathrm{B}} T \kappa^{-1} \tan h^{2}\left(e \psi_{0} / 4 \mathrm{k}_{\mathrm{B}} T\right) \exp (-\kappa D)-A_{\mathrm{H}} / 6 D^{2}$

where $c$ is the ion concentration, $\mathrm{k}_{\mathrm{B}}$ and $T$ are the Boltzmann constant and absolute temperature, $e$ is the electronic charge, $\psi_{0}$ is the potential at each surface, $\kappa^{-1}=\left(\varepsilon \mathrm{k}_{\mathrm{B}} T / 8 \pi e^{2} c\right)^{1 / 2}$ is the so-called Debye screening length $(\varepsilon$ being the dielectric constant of the water). The first term on the right hand of Eq. (1) is the repulsion due to the electrostatic double layer, while the second term is the van der Waals attraction, with $\mathrm{AH}_{\mathrm{H}}$ being the Hamaker constant.

While the long-ranged equilibrium forces between surfaces across water have long been understood (though with some recent surprises [51]), the dynamic properties of water in ultra-thin confined films were, until recently, less well known. For the case of organic liquids or oils, it has been realized for some time that when confined to molecularly-thin layers (in the range 5-10 molecular diameters or less) such liquids become solid-like, and their effective viscosity diverges $[39,40,52]$. The case of water, however, is very different: In 2001 it was shown that water, even when confined to layers of thickness less than $3 \mathrm{~nm}$, retains its bulk-like fluidity or close to it [37]. The experiments that showed this were based on analysing the jump into adhesive contact from $D=D_{\mathrm{j}} \approx 4 \mathrm{~nm}$ such as shown in the inset to Fig. 1 . An approximate expression was derived for the jump time $\tau_{\mathrm{j}}$ from $D_{\mathrm{j}}$ to $D=0$ :

$$
\tau_{\mathrm{j}}=\left(18 \pi R \eta_{\mathrm{eff}} D_{\mathrm{j}}^{2} / A_{\mathrm{H}}\right)
$$

where $\eta_{\text {eff }}$ is the effective viscosity of the thin liquid film across which the surfaces jump into contact. Estimates of the jump time $\tau_{\mathrm{j}}$ revealed that, within confined films of thickness $<$ ca. $3 \mathrm{~nm}, \eta_{\text {eff }}$ was indeed within a factor 3 or so of the value of the bulk 
viscosity $\eta_{\text {water }}$ of water, $\eta_{\text {water }} \approx 0.001 \mathrm{~Pa} \cdot \mathrm{s}$. Why does water in such ultra-thin films behave so differently to oils or organic solvents which solidify under such confinements? The answer is illustrated in Fig. 2.

This shows schematically the disposition of the molecules of a liquid confined between two surfaces. The van der Waals attraction between the molecules and the surface results in a densification of the molecules in the immediate vicinity, within $\sim h$ of the wall (as in Fig. 2), relative to their density in the bulk of the fluid. As the surfaces approach, the two densified regions eventually overlap $(D<2 h)$, so that the overall density in the gap will be larger than that of bulk liquid, and may even exceed that of the solid phase (as shown explicitly in computer simulation studies of alkane liquids [53]). For most nonassociating liquids, including organics and oils, the solid phase is denser than the liquid phase, so that densification of such a liquid in the gap promotes its tendency to solidify. In contrast, for the case of water, almost uniquely, the liquid phase is denser than the solid phase (which is why ice floats). Thus the densification of a thin confined water film acts to suppress its tendency to solidify [54], which is why water in films of thickness down to a single monolayer

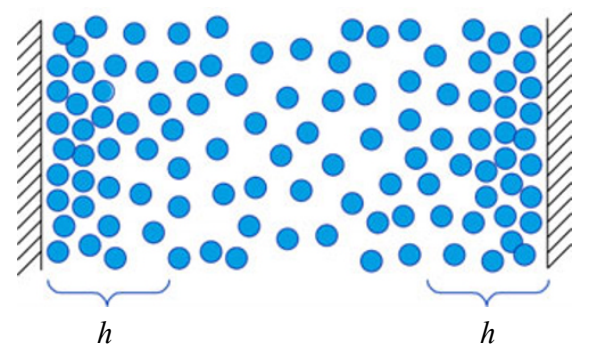

Fig. 2 Schematic illustration of why confined water remains fluid in contrast to organic solvents or oils: When the confined film thickness $D<$ ca. $2 h$, the liquid density in the gap exceeds that of the bulk liquid (and may indeed exceed that of the solid phase [53]), suppressing the tendency of water to solidify, but promoting the solidification of most other liquids. or so retains close to its bulk fluidity.

\section{Water in hydration layers-hydration repulsion}

The water molecule, $\mathrm{H}_{2} \mathrm{O}$, is overall neutral, but possesses a large electric dipole by virtue of the residual charges on the $\mathrm{H}$ and $\mathrm{O}$ atoms, as illustrated in Fig. 3.

As a result of this dipole, water molecules surround charges in aqueous media such as ions or zwitterions (see later) to form so-called hydration layers, as indicated in Fig. 3 for a simple monovalent cation. Such a hydration layer greatly reduces the self-energy (or Born-energy) of the enclosed charge (which may be viewed as the energy associated with assembling the charge). This in turn means that it can be very difficult to permanently remove a water molecule from the hydration sheath surrounding a charge: this is manifested as a large energy of dehydration, as shown in Table 1 below which gives the characteristics of some simple hydrated monovalent ions.

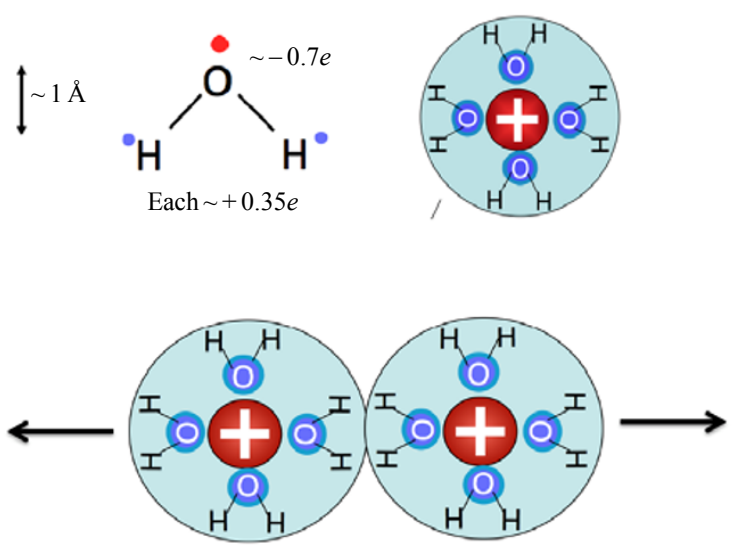

Fig. 3 Illustrating the large dipole of water, and its formation of hydration shells about charges. Bottom: Adjacent charges experience a repulsive interaction of steric origin when their hydration shells overlap.

Table 1 Size and hydration of alkali metal ions.

\begin{tabular}{cccccc}
\hline Ion & $\begin{array}{c}\text { Ionic diameter } \\
(\mathrm{nm})\end{array}$ & $\begin{array}{c}\text { Hydrated ion diameter } \\
(\mathrm{nm})\end{array}$ & $\begin{array}{c}n_{\text {hyd }}, \text { water in } 1^{\text {st }} \\
\text { hydration shell }\end{array}$ & $\begin{array}{c}\text { Hydration energy } \\
\Delta G_{\text {hyd }}(\mathrm{kJ} / \mathrm{mol})\end{array}$ & $\begin{array}{c}\Delta G_{\mathrm{hyd}} / n_{\text {hyd }} \\
(\mathrm{kJ} / \mathrm{mol})\end{array}$ \\
\hline $\mathrm{Li}^{+}$ & 0.12 & 0.76 & $4-6$ & -510 & $-(85-128)$ \\
$\mathrm{Na}^{+}$ & 0.19 & 0.72 & $4-7$ & -410 & $-(59-103)$ \\
$\mathrm{K}^{+}$ & 0.266 & 0.66 & $5-10$ & -337 & $-(34-67)$ \\
$\mathrm{Cs}^{+}$ & 0.338 & 0.66 & $6-12$ & -283 & $-(24-47)$ \\
$-\mathrm{O}-(\mathrm{PEO})$ & & & $(1)$ & -34 & -34 \\
\hline
\end{tabular}

(Adapted from Table 2 of Ref. [68]) 
The large energies associated with hydration of charges lead to hydration repulsion effects [55-60], as qualitatively illustrated in Fig. 3, lower cartoon: the large energy associated with charge hydration effectively results in a strong, short-ranged repulsion of steric origin as water molecules are attracted into the gap between them to hydrate the charges. It is important to emphasize that the picture of hydration shells in Fig. 3 is very schematic: The actual scenario is highly dynamic, with the directions of dipoles and positions of the water molecules fluctuating rapidly [61]. It is only on a time average that the dipoles point towards the enclosed charge, as indicated, and that one may speak of a given number of water molecules that are associated with the hydration shell, as indicated in Table 1. In addition, exchange of water molecules in the hydration shell with "free" water molecules in the surrounding can occur very rapidly, over times scales $\tau_{\text {exchange }}$ of order $10^{-9} \mathrm{~s}$ for the alkali metal ions in Table 1 [41]. This may also be taken as the relaxation time of the hydration shell, corresponding to relaxation rates $\omega_{\text {relaxation }}=\left(1 / \tau_{\text {exchange }}\right) \approx$ $10^{9} \mathrm{~s}^{-1}$ for the case of the ions in Table 1. These very rapid relaxation times, which are only 100 times longer than the relaxation times (ca. $10^{-11} \mathrm{~s}$ ) of water molecules in the bulk, are an important ingredient of the hydration lubrication mechanism described below. Other ions, especially divalent and trivalent ions [41, 62], may have much longer $\tau_{\text {exchange }}$ times; for example, for the $\mathrm{Cr}^{3+}$ ion, $\tau_{\text {exchange }} \approx O\left(10^{5}\right) \mathrm{s}$, which is some 14 orders of magnitude longer than for the alkali metal ions.

The same hydration effect can also lead to strong short-ranged repulsion when two charged surfaces interact across a high-salt-concentration solution $[55,59]$. When such surfaces interact across pure or low salt-concentration water, as shown in Fig. 1, the counterions in the gap are mostly small hydrated protons which, as the surfaces approach, may readily condense into, and neutralise, the negatively charged lattice sites, so they come into adhesive van der Waals contact. In contrast, interactions across high salt solutions, above some critical hydration concentration (which differs with the ion but for $\mathrm{Na}^{+}$, $\mathrm{K}^{+}$is around $\left.0.1-1.0 \mathrm{mM}[57,59]\right)$ may be very different, as illustrated in Fig. 4 for the case of $0.1 \mathrm{M}$

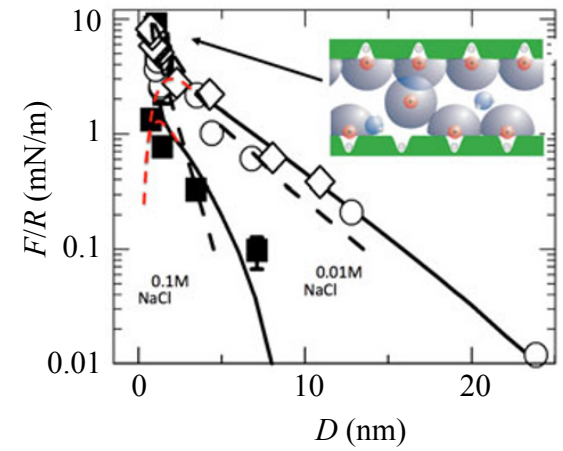

Fig. 4 Normalized force vs. separation $(D)$ profiles between charged mica surfaces in $\mathrm{NaCl}$ solution at two concentrations above the critical hydration concentration. The red broken lines show the profile predicted by theory (Eq. 1 in text) where van der Waals attraction should dominate, while the data shows the strong repulsion actually observed at $D<$ ca. $2 \mathrm{~nm}$, due to hydration effects indicated in the inset cartoon. (adapted from Ref. [45])

\section{$\mathrm{NaCl}$ solution.}

In such a case the counterions trapped between the oppositely-charged surfaces are predominantly hydrated cations (positively-charged $\mathrm{Na}^{+}$ions in Fig. 4). The strongly held hydration layers surrounding each ion result in strong, short-ranged $\left(D<D_{\text {hyd }} \approx\right.$ $2 \mathrm{~nm}$ ) hydration repulsion, which overcomes the van der Waals attraction between the surfaces. This is seen clearly in the force profile, of Fig. 4, where the broken red curves indicate the van der Waals attraction predicted to dominate at $D<$ ca. $3 \mathrm{~nm}$ in Eq. (1), while the data points show the dominant hydration repulsion actually observed. We emphasize that there are two different "trapping" effects which lead to the hydration repulsion: on the one hand, the charges on the surface trap the counterions since the overall charge - surface charge and counterion charge in the gap - must be electroneutral or very nearly so. On the other hand, these trapped counterions in turn hold on strongly to their surrounding hydration water as explained above, which in turn results in the hydration repulsion preventing contact of the surfaces.

We may make a very crude estimate of the maximal pressures $P_{\text {hyd,max }}$ we might expect this hydration repulsion effect to sustain, that is, the pressures up to which the surface interactions are still in the hydration repulsion regime and do not 
adhere. For this we may use values for the "hydration" energy, the energy to permanently remove a water molecule from the hydration sheath, taken from Table 1, for say $\mathrm{Na}^{+}$ions, trapped between mica sheets in high salt solution, as for the system in Fig. 4. As the surfaces approach and enter the range of the hydration repulsion, say at $D<D_{\text {hyd }} \approx 2 \mathrm{~nm}$, further compression is resisted by the reluctance of the hydration water-which is strongly attached to the enclosed ion-to be squeezed out of the gap. To remove all $n_{\text {hyd }}$ hydration molecules $\left(n_{\text {hyd }} \approx 5\right.$ for $\mathrm{Na}^{+}$, from Table 1) requires an energy equivalent to the hydration energy $\Delta G_{\text {hyd }}$, which from Table 1 is $\Delta G_{\text {hyd }} \approx$ $400 \mathrm{~kJ} / \mathrm{mol} \approx 7 \times 10^{-19} \mathrm{~J} /$ ion. If there are $\sigma_{\mathrm{Na}^{+}}$ions trapped/unit area, the total energy/unit area to "dehydrate" them, and so overcome the hydration repulsion, is $\sigma_{\mathrm{Na}^{+}} \Delta G_{\mathrm{hyd}}$. Assuming this energy is provided solely by the work done in compressing the surfaces, we have

$$
\sigma_{\mathrm{Na}^{+}} \Delta G_{\text {hyd }} \approx \int_{D_{\text {hyd }}}^{D_{0}} P_{\text {hyd }} \mathrm{d} D
$$

where $P_{\text {hyd }}$ is the pressure in the hydration repulsion regime and $D_{0}$ is the surface separation at which the hydration shell has been removed by the compression. $P_{\text {hyd }}(D)$ is not precisely known over the range $\left(D_{\text {hyd }}, D_{0}\right)$, though there are indications that it increases exponentially with decreasing $D$ [57]. We therefore make the further approximation that the integral in Eq. 3 is dominated by $P_{\text {hyd }}(D) \approx P_{\text {hyd,max }}$ acting over the last say $\delta D=0.3 \mathrm{~nm}$ of the range, where $\delta D$ is roughly the size of a water molecule. Following this cascade of bold assumptions, we have $P_{\text {hyd,max }} \approx\left(\sigma_{\mathrm{Na}^{+}} \Delta G_{\text {hyd }} / \delta D\right)$. Taking $\sigma_{\mathrm{Na}^{+}}=2 \sigma_{-}$, where $\sigma_{-} \approx$ $1 /\left(0.5 \mathrm{~nm}^{2}\right)$ is the negative-charge surface density on the mica (since both surfaces contribute to $\sigma_{\mathrm{Na}^{+}}$), we obtain finally $P_{\text {hyd,max }} \approx 10^{9} \mathrm{~N} / \mathrm{m}^{2}(=1 \mathrm{GPa})$. This is very likely to be an overestimate, as we neglected the van der Waals adhesive forces between the surfaces as well as the electrostatic energy changes associated with the approach of the $\mathrm{Na}^{+}$ions to the negatively charged lattice sites. But it is probably within an order of magnitude or so of the maximal pressure that the hydration repulsion would support for the system shown in Fig. 4. We note that the data in Fig. 4 shows the repulsion only up to mean pressures $P \approx 0.3 \mathrm{MPa}=3 \times 10^{5} \mathrm{~N} / \mathrm{m}^{2}$, though in very recent work
(Ma et al., to be published, Gaisinskaya et al., to be published) reversible force distance profiles in the hydration repulsion regime between charged mica surfaces (which are essentially a model, atomically smooth solid substrate) across $0.1 \mathrm{M}$ alkali metal salt solutions $\left(\mathrm{Na}^{+}, \mathrm{K}^{+}\right.$ions) have been measured up to $P \approx 10 \mathrm{MPa}=10^{7} \mathrm{~N} / \mathrm{m}^{2}$ (with the hydration lubrication mechanism, see below, acting reversibly up to these pressures). Hydration repulsion forces in a completely different configuration-though one of relevance to systems we describe later in this review - have been measured up to similar pressures $\left(10^{7} \mathrm{~N} / \mathrm{m}^{2}\right)$ between charged lipid bilayers across water [63].

\section{Lubrication by hydrated ions}

From the previous section we see that the hydration repulsion effect arising from trapped, hydrated counterions can support very large pressures while overcoming the van der Waals attraction between surfaces. What of the shear properties of such confined hydrated ions? In their SFB study in 2002, where the underlying ideas of the hydration lubrication mechanism were first formulated, Raviv and Klein [45] measured the frictional forces between mica surfaces in high concentration salt solutions in the hydration repulsion regime (though only, as noted earlier, to mean pressures $P \approx 0.3 \mathrm{MPa}$ ), first revealing the hydration lubrication mechanism. This was later repeated and extended in several studies [64-68] (as well as Ma et al., to be published, Gaisinskaya et al., to be published), and in other measurements where hydration lubrication was implicated [69-71]. Figure 5(a) reproduces the original Raviv and Klein results for $\mathrm{NaCl}$ solutions, while Fig. 5(b) shows results due to Chai et al. [68], who extended these results to other alkali metal ions, illustrating the typical hydration lubrication effect.

The top trace a in Figs. 5(a) and 5(b) is the back and forth motion of the upper surface in the SFB (inset in Fig. 1), sliding across the lower surface. Subsequent traces, taken directly from the SFB, show the friction transmitted across the gap to the lower surface, for different surface separations. Trace b in Fig. 5(a) shows the shear forces when the surfaces are very far apart, 

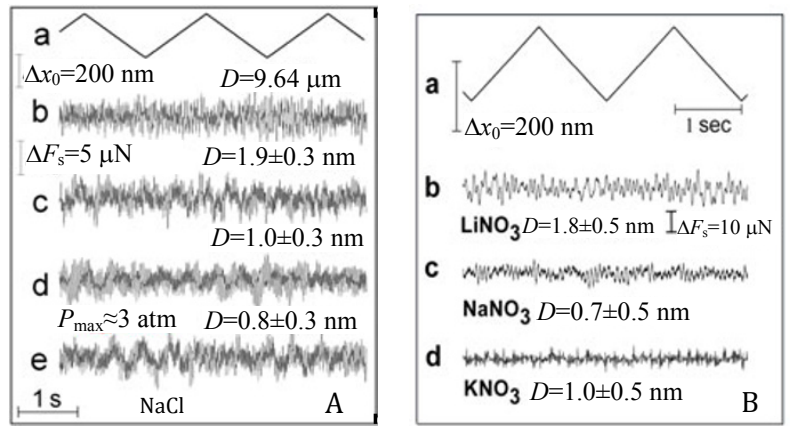

Fig. 5 Typical friction force traces between surfaces sliding across each other in the SFB (inset to Fig. 1) in $0.1 \mathrm{M}$ solutions of alkali metal salts. In both A (top) and B (bottom) traces (a) are the back-and-forth lateral motion $\Delta x_{0}$ of the top surface, while the other traces show the frictional force transmitted to the bottom surface, at separations $D$ as shown, within the hydration repulsion regime. In all cases the friction is within the noise level in the signal. (adapted from Refs. [45] and [68])

establishing the baseline signal for the (essentially) total absence of frictional forces. All other traces in Figs. 5(a) and 5(b) are for $D<2 \mathrm{~nm}$, i.e., in the strongly repulsive hydration regime (see Fig. 4). Clearly the frictional forces in this regime are extremely weak, indeed within the instrumental scatter. An estimate suggests that in these conditions the effective friction coefficient $\mu$ (within the range of sliding velocities and surface pressures studied) is not greater than $\mu \approx$ 0.0002 . This remarkably low value is characteristic of hydrodynamic rather than boundary lubrication, though the conditions of the measurements in Fig. 5particularly the limitingly low sliding velocities - are typical of the boundary lubrication regime. What then is the origin of this strikingly low friction?

The basic idea, which was briefly noted in the introduction, is illustrated in Fig. 6, which shows (roughly to scale) two mica surfaces across a thin $(1 \mathrm{~nm})$ layer of aqueous salt solution (the ion sizes correspond to $\mathrm{Na}^{+}$ions and their first hydration shells, see Table 1 ).

As the top surface slides past the bottom one at velocity $v_{\mathrm{s}}$, the idea proposed by Raviv and Klein [38] was that, as long as the shear rate $\dot{\gamma}=\left(v_{s} / D\right)$ is lower than the relaxation rate $\omega_{\text {relaxation }}=\left(1 / \tau_{\text {exchange }}\right)$ of the hydration shells, the hydrated ions would respond to shear in a liquid-like fashion. At the same time, the hydration repulsion described above acts to keep the surfaces apart even up to high pressures: It is to this

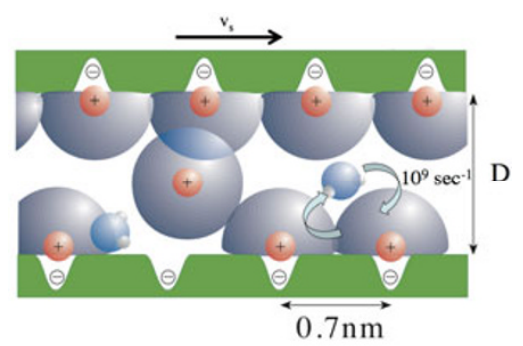

Fig. 6 Illustrating the mechanism of hydration lubrication between charged surfaces across trapped hydrated ions, roughly to scale for mica surfaces and trapped hydrated $\mathrm{Na}^{+}$ions. A large load can be supported by the hydration repulsion (see Fig. 4), but as long as the shear rate $\left(v_{\mathrm{s}} / D\right)$ is less than the relaxation/ exchange rate $\omega_{\text {relaxation }}\left(\approx 10^{9} \mathrm{~s}^{-1}\right.$ for hydration shells on $\left.\mathrm{Na}^{+}\right)$the confined liquid behaves in a fluid manner.

combination-sustaining a high normal pressure and behaving as a fluid under shear-that the very low value of the friction arising from the hydration lubrication mechanism was attributed. A simple estimate of the shear forces based on this idea expected in the Raviv \& Klein experiments bears this out. In those experiments, where sliding velocities $v_{\mathrm{s}}$ reached ca. $1000 \mathrm{~nm} / \mathrm{s}$ and the surface separation in the hydration repulsion regime was around $1 \mathrm{~nm}$ (Fig. 4), the shear rates were of order $\dot{\gamma}=\left(v_{s} / D\right)=$ $10^{3} \mathrm{~s}^{-1}$. Assuming Newtonian behaviour of the liquid in the gap, one expects a shear stress $\sigma_{\mathrm{s}}$ over the flattened contact region, of area $A$, between the compressed surfaces given by $\sigma_{\mathrm{s}}=\dot{\gamma} \eta_{\text {eff }}$, where $\eta_{\text {eff }}$ is the effective viscosity of the liquid (hydrated ions + water) in the gap. The frictional force is then $F_{\mathrm{s}}=A \sigma_{\mathrm{s}}=A \dot{\gamma} \eta_{\text {eff }}$. From the experiments, at the highest load applied, $A \approx 3 \times 10^{-10} \mathrm{~m}^{2}$, while the normal forces (see Fig. 4) go up to $F_{\mathrm{n}} \approx 10^{-4} \mathrm{~N}$. The value of $\eta_{\text {eff }}$ is not directly known. It may be assumed to lie between that of bulk water, $\eta_{\mathrm{H}_{2} \mathrm{O}}$ and the effective "viscosity" of the hydration layers, which may be estimated to be 100 times higher than bulk water since the relaxation times of water of hydration is some 100 times longer than that of water molecules in the bulk. Taking the higher value as an upper limit, $\eta_{\mathrm{eff}}=100 \eta_{\mathrm{H} 2 \mathrm{O}} \approx 0.1$ Pa.s, and the values of $A, \dot{\gamma}$ and $F_{\text {n- }}$ above, allows us to estimate an effective friction coefficient $\mu=F_{\mathrm{s}} / F_{\mathrm{n}}=A \dot{\gamma} \eta_{\mathrm{eff}} / F_{\mathrm{n}} \approx 3 \times 10^{-8} / 10^{-4}=0.0003$. This estimated value, which is an upper limit, compares with the upper limit estimate $\mu \approx 0.0002$ made in the Raviv \& Klein study [38] based on the 
shear force traces.

Very recently, SFB experiments by Ma et al. (to be published) examined the velocity dependence of the frictional forces between the mica surfaces (in $\mathrm{Na}^{+}$ solutions) at shear rates and loads up to 2 orders of magnitude larger than in the original Raviv \& Klein study. These indicate that the simple picture suggested above may be more nuanced, at least for the case of hydration lubrication by trapped hydrated counterions as shown in Fig. 6. However, the underlying framework - of a "solid-like" response to normal load due to the hydration repulsion, together with a fluid response to shear due to rapid relaxation of hydration layers - remains the essence of the hydration lubrication mechanism, and is supported also by detailed molecular dynamics simulations [61].

While the most comprehensive studies on lubrication by hydrated ions come from studies on interactions between atomically-smooth, sliding mica surfaces, recent work has extended this also to a somewhat rougher, metallic surface. Chai \& Klein created extremely smooth (though not atomicallysmooth) gold surfaces via template stripping [72], which could be used as one of the interacting substrates in the SFB [73]. In Fig. 7 the normal interactions between a mica surface and such a gold surface are shown, both in pure water and in a high concentration salt solution $\left(0.01 \mathrm{M} \mathrm{KClO}_{4}\right)$.

The pure water profile between mica and gold is very similar to the mica-mica profile, including the

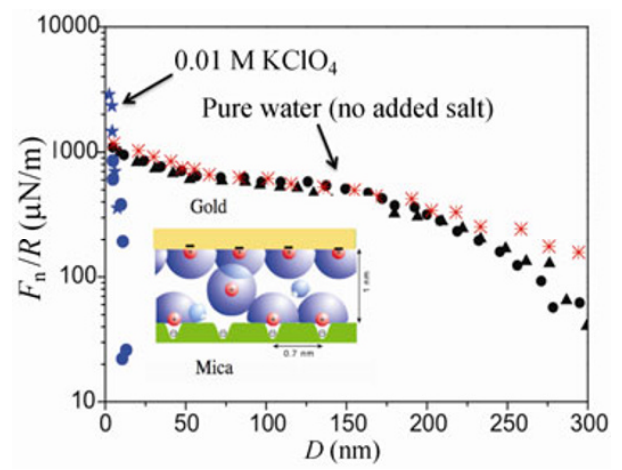

Fig. 7 Force distance profiles between a mica surface and a gold surface (cartoon inset), across water with no added salt, and at high salt concentration in the hydration repulsion regime, as shown by the blue data points. (adapted from Ref. [73]) jump-in to contact from a few nanometer separation (see also Fig. 1), indicating that the gold surface is negatively charged (likely by an excess of $-\mathrm{OH}^{-}$ions) with a charge density very similar to that of the mica. The profile at high salt-blue symbols in Fig. 7shows clearly the hydration repulsion as hydrated $\mathrm{K}^{+}$ counterions are trapped between the negatively charged gold and mica surfaces. Figure 8 shows the shear force traces for this system: trace a shows the lateral motion applied to the top surface, while traces (b)-(f) show the shear forces transmitted to the lower surface.

As in Fig. 5, the friction forces are extremely weak, indeed within the experimental scatter, indicating lubrication by the hydrated trapped $\mathrm{K}^{+}$ions. The slightly higher friction seen in trace $\mathrm{f}$ at relatively low pressures is likely to arise from asperity contacts between the gold-which is not atomically smooth as the mica is-and the opposing mica surface, rather than from breakdown of the hydrationlubrication mechanism. This observation of such low friction also at a sliding metal surface indicates the generality of the hydration lubrication effect, and that one does not require atomically smooth mica surfaces for this mechanism to operate.

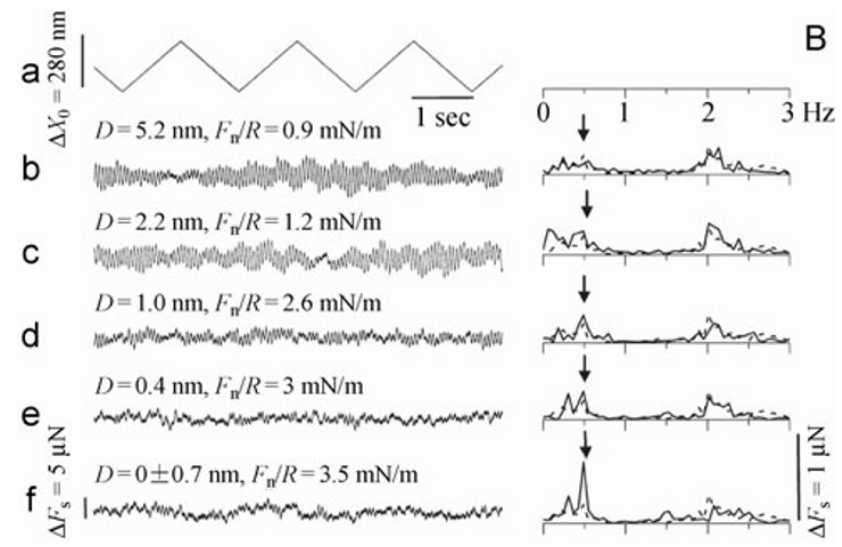

Fig. 8 Friction force traces between mica sliding on gold in $\mathrm{KClO}_{4}$ solution in the hydration repulsion regime (Fig. 7). Top trace (a) is the applied lateral motion of the top surface, while other traces show the friction transmitted to the lower surface at increasing loads (decreasing $D$ ). The $\mathrm{RH}$ traces are a frequency analysis of the friction force (arrows indicate drive frequency) showing little friction above the noise level except for the bottom trace. (adapted from Ref. [73]) 


\section{Lubrication by hydrated polymer brushes}

Already in 1994 it was shown [21] that neutral polymer brushes in an organic solvent could act as extremely efficient boundary lubricants when attached to opposing surfaces as they slid past each other, up to moderate compressions (ca. $3 \mathrm{~atm}$ in the case studied). This occurs because, as a result of entropic factors, the opposing brush molecules interpenetrate only very weakly when compressed $[20,26,27]$ : It is energetically favourable for two swollen brushes on initial compression to become less swollen and denser than to interpenetrate each other. It may be shown $[20,27]$ that the thickness $d$ of the overlapped region of the two brushes in the moderate compression regime varies only very weakly with the compression:

$$
d \sim D^{-1 / 3}
$$

where $D$ is the separation between the substrates. This means, for example, that an 8-fold compression of the brushes under a normal load which increases the polymer segment concentration $c$ in the gap by a similar factor will only double the extent of the overlapped or interpenetrated zone. For this reason the interpenetration zone between the polymer brushes, which is where viscous dissipation occurs on sliding, remains unentangled up to moderate compressions and thus quite fluid. At the same time the osmotic pressure $\Pi$ of the polymer segments, which varies approximately as $\Pi \sim c^{2}$, can support a large external load. Already at the time it was conjectured that this so-called "entropic lubrication" might play a role also in biological systems, where flexible macromolecules are ubiquitous at interfaces between sliding surfaces, such as eyes or joints. Since in biology the medium is water rather than organic solvents or oils, and since most biological macromolecules are in part ionized or polar and so to some extent hydrated, brushes consisting of charged polymers are clearly a more appropriate model for biological lubrication.

Raviv et al. [44,74] studied the shear forces between charged polymer brushes in water, and discovered that such brushes provided boundary lubrication that was significantly more efficient than neutral polymer brushes. This is shown in Fig. 9, which plots the sliding friction coefficient as a function of polymer volume fraction $\phi$ in the compressed polymer layers; $\phi$ is a measure of the extent of compression of the surface-attached polymer layers.

As indicated by the different data symbols and the cartoons in Fig. 9, the charged brushes provide considerably better lubrication up to higher compression than either neutral brushes-whether in organic or in aqueous media $[75,76]$ - or than adsorbed polyelectrolytes $[77,78]$. This may be understood in terms of the structure and hydration of the charged brushes, as illustrated in the cartoon in Fig. 10.

The cartoon, which shows not only the charged chains themselves but also the mobile counterions trapped within the brush layers (green symbols) illustrates the modes by which charged brushes reduce friction between sliding surfaces. Up to moderate compressions the brushes interpenetrate only weakly $\left(d \sim D^{-1 / 3}\right)$, as is the case for neutral chains and arises from configurational entropy effects as described above. The load is then borne by the osmotic pressure of the polymer segments. An additional factor contributing to this load-bearing is

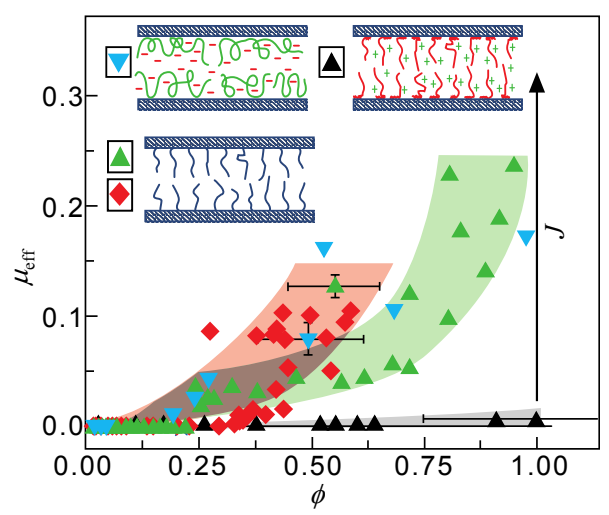

Fig. 9 Variation of the effective friction coefficient $\mu_{\text {eff }}$ between polymer-bearing surfaces with the volume fraction $\phi$ of compressed polymer for neutral brushes, for charged adsorbed polymers and for charged brushes, as indicated by the cartoons and symbols. At the point $J$ for charged brushes the polymer is sheared off the surfaces and the friction rises abruptly. (adapted from Ref. [44]) 


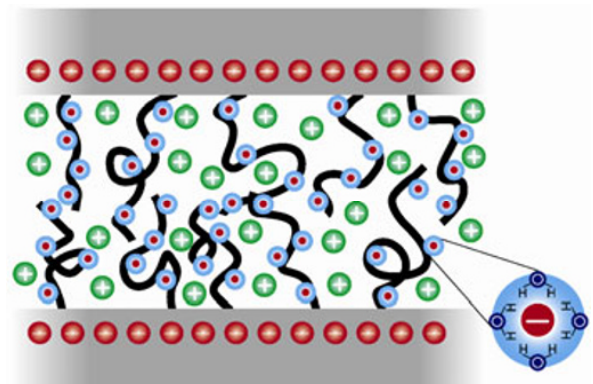

Fig. 10 Illustrating schematically the origin of the lubrication by charged brushes. Up to moderate compressions there is little interpenetration between brushes, resulting in an unentangled overlapped layer and so little viscous dissipation. At higher compressions the hydration layers on the monomers act via the hydration lubrication mechanism. The mobile counterions (green circles) augment the osmotic pressure of the polymer segments and help to support the load.

the osmotic pressure of the trapped counterions themselves, although they are not likely to provide lubrication as such. At the highest compressions, however, when the surface separation becomes comparable with or smaller than the radius of gyration of the polymer chains $\left(D<\mathrm{ca} . R_{\mathrm{g}}\right)$, one expects the entropic effect suppressing the interpenetration to become negligible [20,27]. It is at these higher compressions that lubrication by the hydration layers on the charged monomers, indicated in the cartoon, becomes the dominant lubricating mode via the hydration-lubrication mechanism. Since frictional dissipation takes place across the sheared interpenetration zone [29] rather than at a sharp interface as between solid surfaces, the hydration layers reduce the friction between the compressed monomers as they slide past each other, via the hydration lubrication mechanism. Both the additional load-bearing osmotic pressure due to trapped counterions, and the lubricating effect of the hydration shells about the charged monomers, are effects that do not apply in the case of neutral chains. They do however apply for adsorbed charged polymers: the reason why adsorbed chains (blue triangles in Fig. 9) are far inferior lubricants to the charged brushes is due to bridging effects which occur whenever two adsorbed layers interact. Such bridging occurs when a chain from one adsorbed layer adsorbs also to the opposing surface: When the surfaces slide past each other, the bridging chains are dragged past the surfaces onto which they are adsorbed, leading to energy dissipation and thus to higher friction [30,77,78]. We note that neutral brushes in aqueous media, such as brushes consisting of poly(ethylene oxide), also provide quite weak lubrication $[75,76]$. This is likely to be because the PEO monomers are polar, so that the extent of hydration of their monomers (see also Table 1), and thus the efficiency of the hydration lubrication effect, is expected to be much weaker than for charged (or zwitterionic) monomers. Indeed, the issue of the extent of hydration of charged charged groups, which can differ greatly depending on the particular charged group structure or the surrounding salt concentration, is crucial to understanding and controlling hydration lubrication and is a recurring motif in this review.

In the study on charged brushes by Raviv et al. $[44,74]$, the polymer chains consisted of diblock copolymers, where a hydrophobic block physically attached to the solid (hydrophobized) surfaces, driven by hydrophobic attraction. This is a relatively weak attachment, and indeed it was observed that at quite low pressures, ca. $3 \mathrm{~atm}$, as the monomer density increased, friction between the monomers became large enough to shear the polymer brushes off the sliding surfaces. At this point, with the brushes removed, the surfaces jumped into adhesive contact and the friction coefficient increased abruptly, as indicated by the arrow J in Fig. 9. To overcome this, Chen et al. [79] adopted a different strategy-covalently growing polymers from the substrate to produce a much more strongly attached brush, consisting of poly[2-(Methacryloyloxy)ethyl PhosphoCholine], pMPC, chains [43, 79, 80]. Such grafted-from brushes are not only much more robust with respect to being torn off the surfaces, but in addition, and very importantly, each of the monomers on the pMPC chains had the structure of a phosphocholine group (as indicated in the insets to Fig. 11 and also Fig. 12).

Phosphocholine groups are ubiquitous in biological systems, including especially cell membranes where they form the headgroups of a large class of phospholipids. The phosphocholine group is zwitterionic, i.e., overall neutral but 


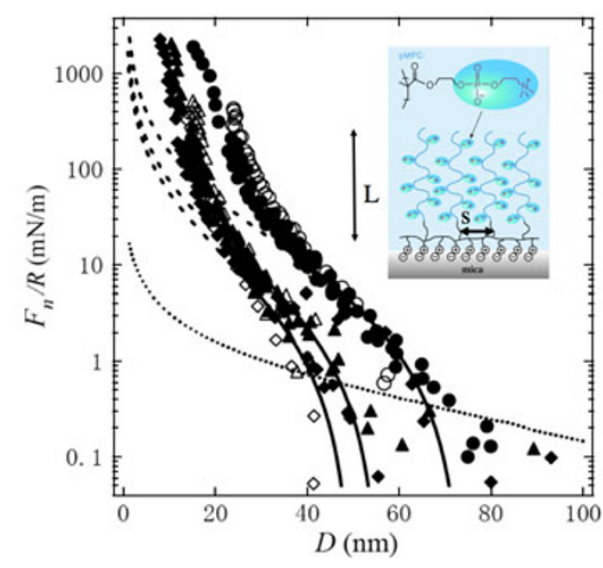

Fig. 11 Force distance profiles between surfaces bearing zwitterionic (pMPC) brushes of structure as indicated in the inset, grown from a macroinitiator layer (inset). The three profiles (lines are fits to the Alexander-de Gennes model which enables extraction of chain height $L$ and spacing $s$ on the surface), in order of decreasing onset of repulsion, are in water (no added salt), $0.01 \mathrm{M}$ and $0.1 \mathrm{M}$ salt concentrations respectively. At the highest loads the mean contact pressure is ca. $8 \mathrm{MPa}$. (dotted line is the interactions between the macroinitiator layers prior to grafting polymer. Adapted from Ref. [43])

containing both negative $\left(\mathrm{PO}_{4}^{-}\right)$and positive $\left(\mathrm{N}^{+}\left(\mathrm{CH}_{3}\right)_{3}\right)$ charged groups; in particular, with respect to lubrication, they are very highly hydrated, with up to 15 or more water molecules in the primary hydration shell (depending on the method used to determine this [81-86]). For comparison, the common alkali metal ions such as $\mathrm{Na}^{+}$and $\mathrm{K}^{+}$, Table 1 , which provide excellent lubrication when trapped between charged sliding surfaces, as shown in Figs. 5 and 6, have fewer than half that number of water molecules in their primary shell. Figure 11 shows the normal force profiles $F_{\mathrm{n}}(D)$ between two pMPC-brush-bearing surfaces, where at the highest loads the pressure across the contact area is ca. 80 atm $(\sim 8 \mathrm{MPa})$. Indicated in the inset is a schematic of the pMPC brush. Because they are covalently grown from the surface, the brush "density", expressed in terms of the ratio $(L / s)$, where $L$ is the brush thickness and $s$ is the mean brush spacing, can reach much higher values than for physisorbed brushes; values of $(L / s) \approx 25$ have been attained (Tairy et al., to be published) compared with $(L / s) \approx 6-8$ for physically attached brushes. The higher $(L / s)$ ratio is associated with reduced relative interpenetration of the chains when the brushes are compressed. The robustness of the brushes, their high level of hydration, and the large $(L / S)$ ratio, should thus all act to improve their lubrication properties.

Chen et al. [80] measured the friction coefficients between sliding surfaces bearing such brushes [43]. The results are summarized in Fig. 12, which shows that the friction coefficients in pure water remain as low as 0.0004 even at pressures as high as ca. $8 \mathrm{MPa}$ (80 atm).

These high pressures are of special interest as they are comparable with the maximal pressures in the major joints such as hips or knees (Indeed, in a study examining the use of polymer brushes as boundary lubricants for prosthetic hip implants [87], a use first proposed by Raviv et al. [44], similar pMPC brushes grafted onto the polythene acetabular cup surface of the implant were found to reduce massively the wear of the material at physiological pressures. The friction was less affected, possibly because of asperity contacts). At physiologically high salt concentrations up to $0.1 \mathrm{M}$ salt, the friction coefficient with the pMPC brushes was found [43] to increase slightly (to around $0.001-0.002)$ : This is probably the result of some dehydration of the phosphocholine monomers by the salt ions (so-called "salting out" effect [88]). The origin of such massive reduction in friction is believed to be the high hydration level of the

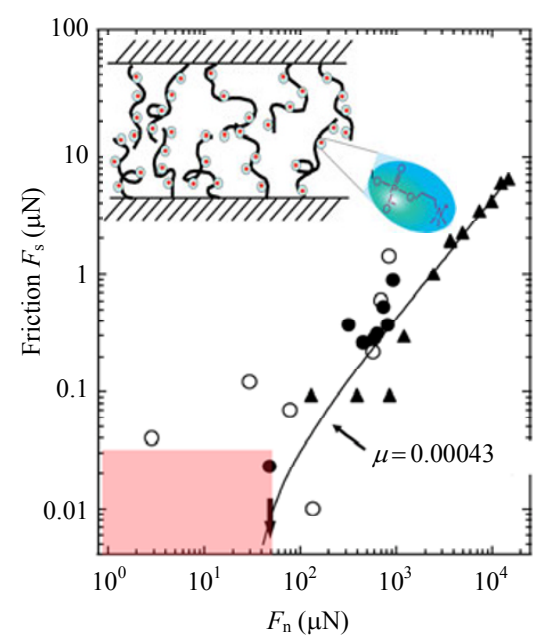

Fig. 12 Variation of friction force $F_{\mathrm{s}}$ with load $F_{\mathrm{n}}$ between surfaces bearing pMPC brushes in water as in Fig. 11, indicating a friction coefficient $\mu$ as shown. The inset illustrates the mechanism: At the highest loads the lubrication is attributed entirely to the hydration layers surrounding the phosphocholine groups. (Adapted from Ref. [43]) 
phosphocholine monomers of the pMPC brushes, as illustrated in the inset to Fig. 12. At moderate compressions the brush-like nature of the layers suppresses their interpenetration as noted above for charged brushes. However, at the very much higher pressures which the chemically-grafted, robust pMPC chains can support, such effects, of configurational entropy origin, become negligible as the intersurface gap $D$ becomes comparable or smaller than $R_{\mathrm{g}}$. In addition, the polymers are zwitterionic, that is, they are overall neutral, in contrast to the charged brushes in Figs. 9 and 10. There are therefore no mobile counterions trapped in the chain layers to enhance the osmotic pressure as for charged brushes. The entire reduction in frictional forces between the zwitterionic brushes as they slide past each other at the highest compressions may thus be attributed to the hydration shells surrounding the phosphocholine monomers, acting via the hydration lubrication mechanism. We note also more recent macroscopic friction studies $[89,90]$ on pMPC and other hydrated brushes, where higher pressures still were accessed, but where the friction coefficient was substantially higher, of order $0.01-0.05$. This may be in part because at these pressures-about an order of magnitude higher than in the Chen et al. study - more water was squeezed out of the hydrated monomers, thereby weakening the hydration lubrication effect.

\section{Boundary lubrication by amphiphilic surfactants under water}

Boundary lubrication by surfactants was first described by William Hardy early in the 20th century $[10,11]$. In his classical picture, the two solid substrate surfaces sliding past each other were each coated with a monolayer of a surfactant consisting of a polar headgroup and an alkyl tail. The headgroups adhered to the substrate, while the tails were exposed as a close packed layer, and two such boundary layers rubbed past each other as the surfaces slid. Due to the relatively weak shear strength of the van der Waals bonds between them, these layers slid with quite low friction $(\mu \approx 0.05-$ $0.10)$; in particular they protected the underlying substrates (often metals) from contact and wear. This picture holds in air and in organic solvents (such as oils), but under water the behaviour turns out to be remarkably different. Briscoe et al., using an SFB, investigated the behaviour of boundary layers consisting of such amphiphilic surfactants both in air and when immersed in water $[42,91]$. Their essential findings are shown in Fig. 13.

The top trace A in Fig. 13 shows, as in earlier figures, the applied back-and-forth lateral motion applied to the top surface of the SFB, with the surfaces each bearing a monolayer of a double-tailed cationic surfactant of structure $\left[\mathrm{CH}_{3}\left(\mathrm{CH}_{2}\right)_{10}\right]_{2} \mathrm{~N}^{+}\left(\mathrm{CH}_{3}\right)_{2} \mathrm{Br}^{-}$. The positively charged ammonium group attaches to the negatively charged mica surface, while the alkyl tails are exposed. Both the geometry of contact and the thickness of the boundary surfactant layers is revealed by the shape of the interference fringes in the SFB experiment, lower right inset in Fig. 13. In air the two
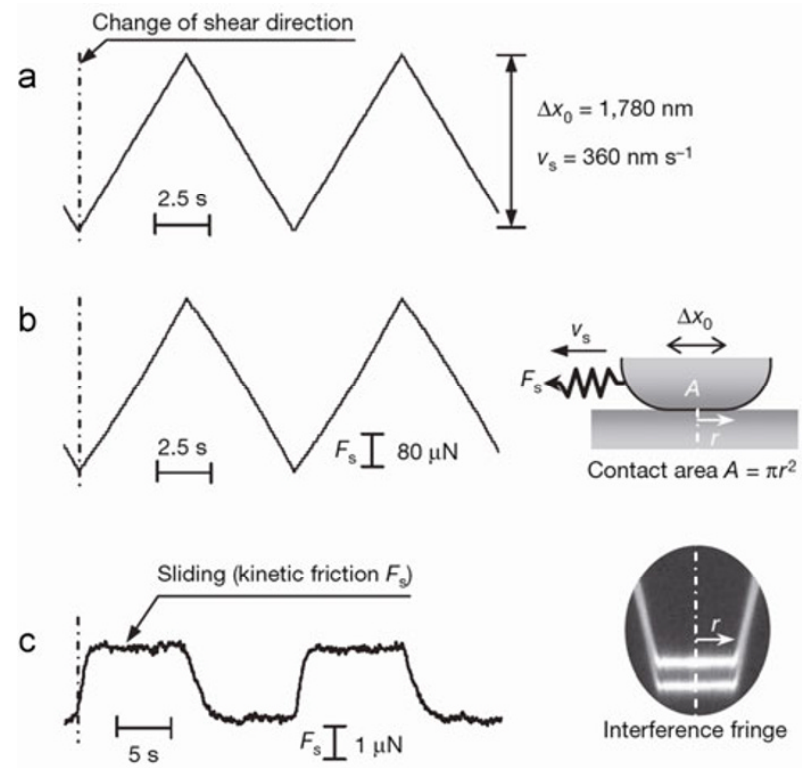

Fig. 13 Friction force traces between mica surfaces bearing surfactant layers. Top trace A is the lateral motion applied to the top surface, centre RH inset. Traces B, C show the shear forces transmitted to the lower surface, showing that in air (trace B) the friction exceeds the maximal applied shear force so that there is no sliding. On adding water, trace $\mathrm{C}$, the friction drops by a factor of at least 100-fold. Lower RH inset shows the interference fringes observed in the SFB, which reveal that on adding water the surfactant layers swell by ca. $2.5 \AA$ each. (adapted from Ref. [42]) 
monolayer-bearing surfaces come into adhesive van der Waals contact over a contact area $A$ (insets to Fig. 13), and the friction $F_{\mathrm{s}}$ between them is larger than can be overcome by the shear forces applied as the top surface is moved laterally. Because of this the surfaces do not slide past each other, but rather move back and forth in tandem, as seen in trace B of Fig. 13. When water is added, however, two striking effects are noted. Firstly, the friction between the surfaces falls by at least two orders of magnitude, as seen in trace $C$ of Fig. 13. Secondly, the interference fringes shift, indicating that each surfactant layer has become thicker by some $0.25 \mathrm{~nm}$. By carrying out further measurements, particularly on the adhesion hysteresis between the surfaces both in air and under water, Briscoe et al. [42] were able to pinpoint the origin of the very large reduction in friction, which is illustrated in Fig. 14.

In air (and likewise in oils), as shown long ago by Hardy and extensively studied since $[7,8,92,93]$, the boundary layers adhere through van der Waals bonding at the interface between their alkyl tails. When sliding past each other, these bonds shear and sliding occurs at the midplane interface, which is then the slip plane. When water is added to the system, however, it penetrates the surfactant monolayers to hydrate the polar headgroup layer at each mica surface, as illustrated in Fig. 14. That is the origin of the $0.25 \mathrm{~nm}$ swelling of each surfactant monolayer on addition of water. These charged,

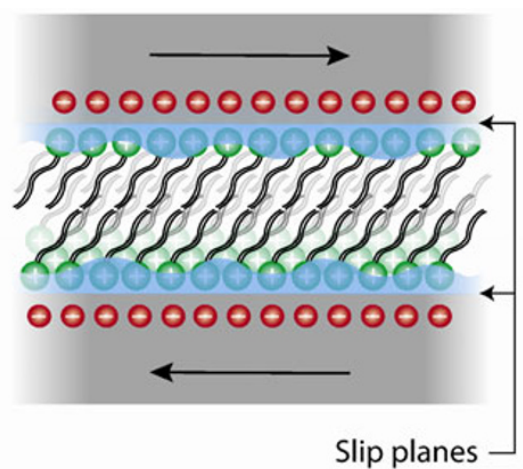

Fig. 14 Schematic illustration of the origin of the large friction reduction between surfaces coated by close-packed surfactant layers as seen [42] in Fig. 13, trace $C$. On adding water, it penetrates and hydrates the head-groups at the mica surfaces, enabling hydration lubrication and the shift of the slip planes to that interface as indicated. hydrated headgroup layers can now slide easily past the solid substrate via the hydration lubrication mechanism, and the slip plane shifts from the mid-plane between the boundary layers to the boundary-layer/substrate interface, as indicated in Fig. 14. Briscoe et al. $[42,91]$ were also able to show that when the surfaces are separated (and then rejoined) they come apart and together again at the mid-plane, but when sliding they slide at the substrate interface. In other words, the two surfaces adhere at one plane, but slide at another. This remarkable behaviour has recently been seen also with other amphiphilic surfactants [94].

\section{Lubrication by liposomes}

The striking lubrication by the hydrated phosphocholine-like monomers of the pMPC brushes (Section 5), together with the very low friction surface provided by an interfacial layer of hydrated surfactant headgroups (Section 6), suggests that phospholipid bilayers could act as efficient boundary lubricants. Indeed, the lubricating properties of such bilayers have been extensively studied (see $[95,96]$ and references therein). In the study by TrunfioSfarghiu et al. [96] they were shown to reduce friction substantially via the hydration lubrication mechanism, though up to relatively low mean pressures (ca. $0.3 \mathrm{MPa}$ ). A different approach is to utilize phospholipid vesicles, known also as liposomes, which are composed of phospholipid bilayers (similar to cell membranes), as illustrated in Fig. 15.

The outer liposome surfaces expose the lipid headgroups; when these are phosphocholine groups, the lipids are known as phosphatidylcholines (PCs). Most PCs are insoluble in water as single molecules, however, and liposomes provide a ready means of introducing PCs into aqueous media. Liposomes have been widely used in medical diagnostics and for drug delivery $[97,98]$, but their use as boundary lubricants on solid surfaces has only very recently been explored. Goldberg et al. [46, 99] deposited small (diameter ca. $70 \mathrm{~nm}$ ) unilamellar (single bilayer wall) vesicles of Hydrogenated Soy PC (HSPC, structure shown in Fig. 15) onto mica. The liposomes 


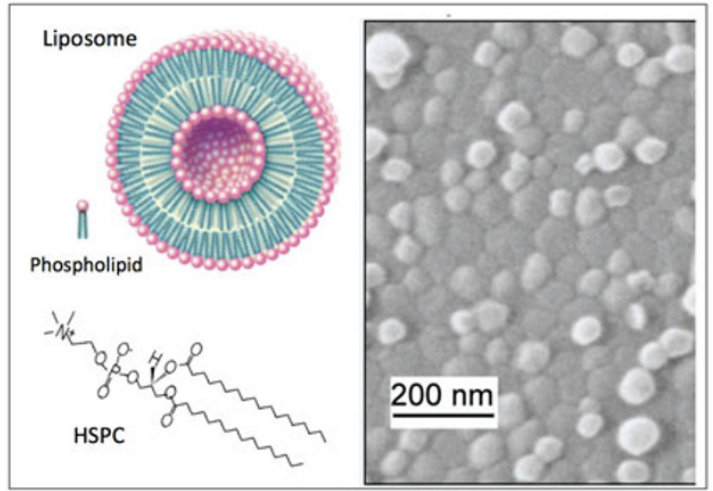

Fig. 15 A cryo-Scanning Electron Microscopy picture of liposomes of hydrogenated soy phosphatidylcholine (HSPC, structure shown) adsorbed onto mica from dispersions. A close packed layer of the liposomes on the mica is covered by a sparser overlayer of loosely attached liposomes (lighter shade). (Micrograph adapted from Ref. [46])

form a close packed monolayer on the mica, as seen in the micrograph in Fig. 15. The friction acting between two such liposome-coated surfaces was measured in the SFB, and the results are shown in Fig. 16.

Extremely low friction coefficients were measured between the sliding surfaces, as low as $\mu=2 \times 10^{-5}$ at mean pressures of over $10 \mathrm{MPa}(100 \mathrm{~atm})$. The origin of this very low friction is again attributed to the

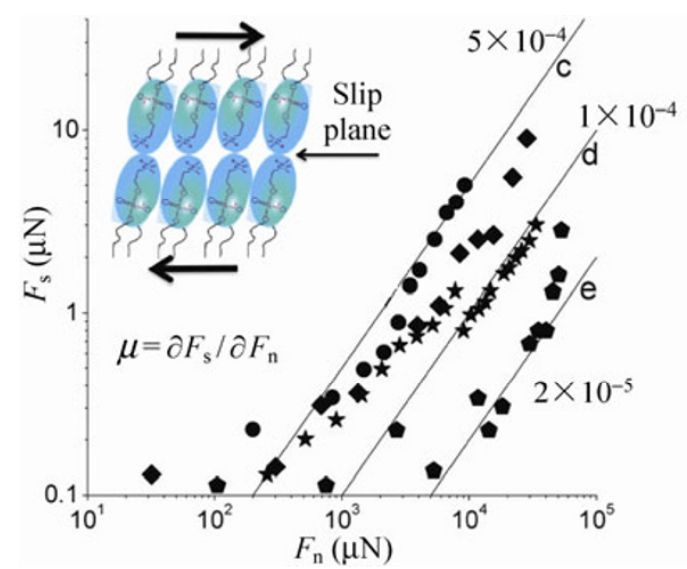

Fig. 16 Variation of friction $F_{\mathrm{s}}$ with load $F_{\mathrm{n}}$ between two HSPC-liposome-bearing surfaces (as in Fig. 15), with the highest loads corresponding to contact pressures of over $10 \mathrm{MPa}$. The lines $c, d, e$ represent friction coefficient values as indicated $(\mu=$ $5 \times 10^{-4}, 1 \times 10^{-4}$ and $2 \times 10^{-5}$ respectively). The inset illustrates the hydration lubrication mechanism as two hydrated phosphocholine head-group layers exposed by the liposomes rub past each other. (adapted from Ref. [46]) hydration lubrication mechanism, as the highlyhydrated phosphocholine layers exposed by each liposome layer rub against each other, as illustrated in the inset to Fig. 16. The robustness of such liposome boundary layers to high pressures and shear was attributed [46] to a number of effects: to the HSPC liposomes being in their solid-ordered phase, in which the bilayers have a more rigid structure; to the closed nature of the PC vesicles themselves; and to their close packed nature on the surfaces. Very recent systematic studies on a series of PC liposomes with different tail lengths (Sorkin et al., to be published) have revealed more subtle effects controlling the robustness of the liposome surface layer (and that merely being in the solid-ordered phase is not sufficient). The extreme boundary lubricating properties of liposomes arising from the hydration lubrication mechanism thus offers great promise for medical and biomedical applications where friction is an issue, including osteoarthritis, contact lenses or knee- or hip-joint implants. This is because such liposomes, often being composed of naturally-occurring and biologically ubiquitous phospholipids, are fully biocompatible.

\section{Hydration lubrication in biological systems}

The natural medium of living systems is water, so that most biological molecules or molecular assemblies-cells, organelles, proteins, macromolecules, connective tissues, components of the extracellaular matrix - are at least partly hydrophilic or expose hydrophilic groups. Such groups may be charged, polar, dipolar or zwitterionic, and as such are likely to be hydrated to a lesser or greater extent. Thus we expect lubrication in biology to be in substantial measure related to the hydration lubrication mechanism, in addition to other frictional dissipation processes. Of particular interest both from a tribological, but also from a biological and biomedical perspective [100], are lubrication processes occurring under conditions of large mechanical stresses, including especially the major joints (hips or knees). 
In such joints, layers of articular cartilage (coating the ends of the articulating bones) rub past each other; the cartilage consists of a network of collagen fibres, permeated with a large array of charged, flexible macromolecules, proteins, phospholipids and other components (see review in Ref. [101]); a schematic of a hip joint is shown in Fig. 17.

The joint cavity is filled with synovial fluid, which also contains a range of flexible, charged macromolecules, proteins, and lipids. From a tribological point of view, the synovial joint is one of the most remarkable constructs in nature. Through the use of implants with pressure sensors, local pressures up to ca. $20 \mathrm{MPa}$ (ca. $200 \mathrm{~atm}$ ) have been measured in hip joints $[102,103]$, while the mean pressure during the peak of a normal walking cycle is ca. $5 \mathrm{MPa}$. At the same time, friction between articular cartilage surfaces in living joints at these pressures is extremely low, even at very low sliding pressures. Values down to around 0.003 have been reported in animal joints in vivo or in cadavers [104-106], although in practice it is extremely difficult to measure such low friction coefficients in living joints. This is because it is almost impossible in a frictional torque measurement (the usual approach used in vivo) to separate the frictional dissipation that arises from distortion of surrounding tissue during joint articulation from that due to cartilagecartilage sliding friction (D. Dowson, private

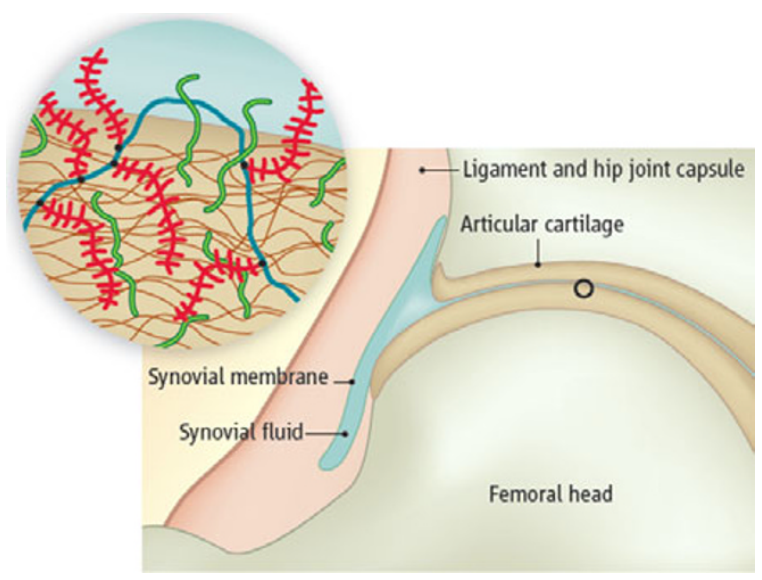

Fig. 17 Schematic of a human hip joint. The inset shows the outer cartilage surfaces with cartoons, roughly to scale, indicating the major macromolecular species: HA (blue), Agg (red bottle-brush-like molecules) and lubricine (green). (adapted from Ref. [36]) communication). For this reason, even the low values reported for friction coefficients from in vivo measurements are likely to give values that are higher than the intrinsic friction coefficient associated with cartilage-cartilage sliding under high pressure. At the same time, once cartilage is removed from the body and examined in physiological saline, say, its surface characteristics change rapidly [107], and friction measurements are unlikely to reproduce the lubrication processes that occur between sliding cartilage surfaces in living joints. Healthy synovial joints in humans, therefore, may be taken to operate over a large range of shear rates (from rest to ca. $10^{6} \mathrm{~s}^{-1}$ ) and loads (pressures up to order $100 \mathrm{~atm}$ or more) with friction coefficients of order 0.001: No man-made joints approach this level of tribological sophistication.

The importance of understanding the mechanism of lubrication that leads to such low friction in healthy joints, which has implications both for clinical and biomedical applications, has spawned a large number of models (see Refs. [100] and [101] for reviews of these). These range from extension of ideas from engineering tribology, to microscopic models based on the known molecular components of cartilage and of synovial fluid. The low friction in joints even at very low sliding velocities and even following extended periods of rest under load, suggest that boundary lubrication at the cartilage surface must play an important role. Several groups have studied the boundary lubrication properties of different molecular and macromolecular species associated with cartilage/synovial fluid [108-117], though a boundary lubrication mechanism duplicating the low in vivo friction coefficient has not yet been identified: The hydration lubrication paradigm that has emerged over the past decade for understanding frictional mechanisms in aqueous media, described in this review, provides a useful framework for such studies.

Very recent attempts to pinpoint the relevance of this mechanism have examined systematically, using an SFB, the boundary lubrication properties of two of the main macromolecular species in cartilage, hyaluronan (sometimes referred to as hyaluronic acid (HA)), a linear polysaccharide, and aggrecan (Agg), a 
bottle-brush-like proteoglycan. In cartilage, these macromolecules are synthesized in the chondrocyte cells native to the cartilage, and complex with each other so that the Aggs attach to the HA backbone: Fig. 17 illustrates the structure schematically. The HA/Agg complex, both of whose components are highly charged, must diffuse after its synthesis in the cells to permeate the cartilage network, where it provides the osmotic pressure that gives this network its mechanical properties. This complex presumably also diffuses to the surface regions of the cartilage which rub against the opposing cartilage surface. It has been conjectured [101] that, being highly charged, the HA/Agg complex might act-much as the charged or zwitterionic brushes described in section 5 above - as an efficient boundary lubricant via the hydration lubrication mechanism. Indeed, HA alone-though originating in the synovial fluid rather than in the cartilage - was long thought to be an important contributor to lubrication of joints (see for example Refs. [118], and also [119]). Injection of HA as a "visco-supplement" for relief of painful joints is to this day a popular clinical treatment, though its benefits (over and above a placebo effect, say) have been questioned [120], and there is little evidence that it acts to reduce the friction in the conditions of articulating joints. In a series of papers, Seror et al. [121, 122] reconstructed both HA and, separately, the HA/agg complex onto the mica surfaces in the SFB, and measured the resulting boundary lubrication, as summarized in Fig. 18.

In these experiments the HA was first attached to the mica. HA is negatively charged, as is the mica, and to induce its attachment the mica was coated with avidin and the HA was functionalized with biotin; biotin forms strong specific attachment to avidin, as indicated schematically in the left inset in Fig. 18. Friction forces as a function of the load, shown as empty triangles and stars in Fig. 18, indicate a rather high friction coefficient $(\approx 0.3)$ between two such HA-coated surfaces already at quite low pressures. A reason for this may be the means of attaching the HA-via biotinylation and attachment to avidin on the substrate surface - which could lead to bridging of the HA, i.e., a given HA molecule may attach to avidin on both the opposing

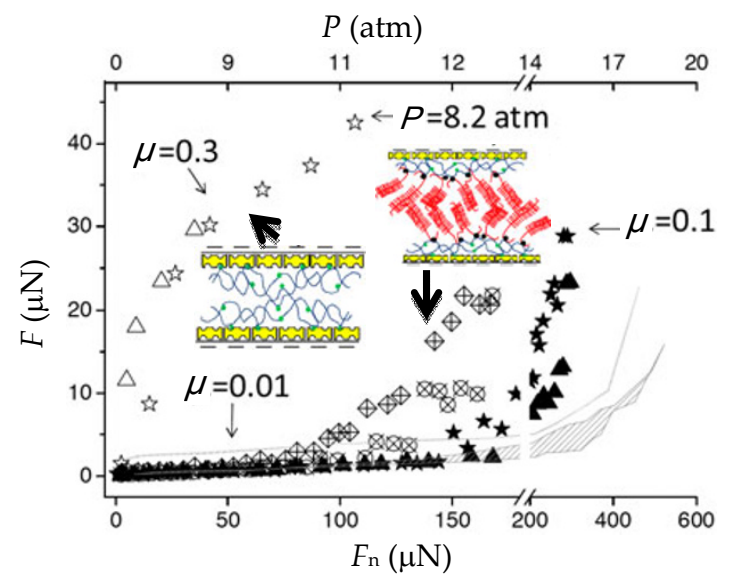

Fig. 18 Friction force $F_{\mathrm{s}}$ vs. load $F_{\mathrm{n}}$ between two surfaces bearing HA layers (empty triangles and stars), LH inset cartoon, and between surfaces bearing $\mathrm{HA} / \mathrm{Agg}$ complexes, $\mathrm{RH}$ inset cartoon. Values of friction coefficients $\mu$ are indicated at different regions of the $F_{\mathrm{s}}\left(F_{\mathrm{n}}\right)$ plots. The upper scale shows the mean contact pressure for the HA/Agg data. (Shaded band is for an HA/Agg complex on mica rubbing against bare mica). (adapted from Ref. [122])

surfaces at the same time, and thus not be representative of the intrinsic friction between HA layers. The relatively poor lubrication is however in line with the conclusions of an earlier study on HA lubrication to much higher pressures [113] and may be due to poor hydration of the charged groups $\left(-\mathrm{COO}^{-}\right)$on the $\mathrm{HA}$, as discussed below. Once Agg molecules are attached to the HA to form complexes similar to those in bulk native cartilage, as indicated by the right cartoon in Fig. 18, the friction coefficient is markedly reduced (to $\mu \approx 0.01$ ) up to ca. $10 \mathrm{~atm}$ pressure. This may be for two reasons: firstly, there is a much smaller likelihood of bridging by the exposed, highly negatively charged Agg molecules to the avidin coating the opposing mica surfaces; secondly, the intrinsic friction between the two Agg layers may be lower due to the much higher charge density on Agg (consisting of $-\mathrm{COO}^{-}$and $-\mathrm{SO}_{3}^{-}$charged groups) relative to HA. At higher pressures-from above ca. $15 \mathrm{~atm}$ in the study by Seror et al.- the friction coefficient increases as shown, possibly due to a greater bridging likelihood. However, even for Agg molecules rubbing against each other at the lower pressures, the friction coefficient is at least ten-fold higher than seen in for articular cartilage. This is thought to be as a result of the relatively poor 
hydration of the negatively charged groups $\left(-\mathrm{COO}^{-}\right.$ and $-\mathrm{SO}_{3}^{-}$) on the Agg molecules.

Another molecule that has been frequently implicated in the boundary lubrication of cartilage is lubricin, an elongated, net negatively charged macromolecule (but bearing both positive and negative charges) present in both cartilage and synovial fluid. Direct measurements with an SFB of the friction between lubricin-bearing surfaces [116] indicate behaviour rather similar to that seen for the HA-Agg complex in Fig. 18: A low friction coefficient $\mu$ (of order 0.02 ) up to ca. 5-10 atm, then much larger $\mu$ values (around 0.2 ) at higher pressures. Thus we conclude that, to date, the major macromolecules (HA, Agg, lubricin) thought to be implicated in cartilage lubrication do not, when measured directly as boundary lubricants in SFB experiments, lead to friction coefficients anywhere near as low as those observed between healthy cartilage at physiological pressures.

In view of the very low friction arising from the hydration lubrication mechanism when phosphocholine groups are implicated, as in Figs. 12 and 16, we note that surface-active phospholipids have been suggested, by Hills and co-workers and by others [123-127], to play a central role in the boundary lubrication of cartilage. However, according to Hills [125] such phospholipids act in the classical boundary lubrication mode: with their phosphocholine headgroups attached to the cartilage surface, and their alkyl tails forming a close-packed layer which slides past a similar opposing alkyl-tail layer. Such a process is known to lead to friction coefficients $\mu \approx$ $0.05-0.1$, which is $1-2$ orders of magnitude greater than in joints, so that the Hills picture is unlikely to be correct.

\section{Conclusions and challenges}

The hydration lubrication mechanism, which differs from the classical modes of lubrication, is a powerful new framework for understanding and controlling boundary lubrication processes in aqueous media. The essential idea is that hydration shells surrounding charged, zwitterionic or polar groups are tenaciously attached, and so able to support large normal stresses without being squeezed out, and at the same time are fluid, so that their sliding past each other or past surfaces can occur at very low shear stresses. This combination provides the elements that can make hydration lubrication extremely efficient, given the right hydrated species and the appropriate vectors to bring them to the desired slip interface. Model experiments between charged, atomicallysmooth surfaces indicate that trapped, hydrated cations (such as the alkali metal ions) can indeed act in this way, but clearly the nature of the hydrated charge plays a crucial role: not all hydrated groups are "equal" in this respect. Thus, in general, we expect anions (negatively charged species) to be less well hydrated than cations [128] and so to be less effective hydration lubrication elements. Polar groups, such as -O- groups on monomers of the neutral but water-soluble polyethylene oxide (PEO), are expected to be even less hydrated (see Table 1) and so less effective still. This might, in particular, account for the breakdown of lubrication at relatively low $P$ (a few atm) with the charged brushes described earlier (Ref. [44] and section 5), where the hydrated species are $-\mathrm{SO}_{3}^{-}$groups, as well as the relatively weak boundary lubrication effect by the $\mathrm{HA}$ and $\mathrm{HA} / \mathrm{Agg}$ complexes, where the hydrated species are $-\mathrm{COO}^{-}$and $-\mathrm{SO}_{3}^{-}$(Ref. [122] and section 8). It would also readily explain why PEO brushes in water $[75,76]$ provide only very moderate lubrication.

Even within a given series of cations such as the alkali metal series $\mathrm{Li}^{+}, \mathrm{Na}^{+}$, etc., however, there may be large differences in the hydration lubrication efficiency depending on the details of the hydration. Thus we know that $\mathrm{Cs}^{+}$, the largest ion in this series, has the most weakly held hydration shell (Table 1), and for this reason it does not succeed as a lubricant at all [129]. The $-\mathrm{N}^{+}\left(\mathrm{CH}_{3}\right)_{2}$ or $-\mathrm{N}^{+}\left(\mathrm{CH}_{3}\right)_{3}$ group, in contrast, appear highly efficient in the hydration lubrication context, as seen when they form, respectively, a surfactant-headgroup surface layer $[42,129]$ (section 6) or as part of the phosphocholine group on vectors such as polymer brushes [43] (Section 5) or liposomes [130] (Section 7).

Many of the findings reviewed here were revealed through SFB experiments, in which hard, 
atomically-smooth mica substrates, either coated by other species or confining counterions between them, slide past each other. It is thus appropriate to ask how representative that is of sliding between soft surfaces, or between hard surfaces that do not possess mica's single-crystal-plane smoothness (though some indication for the smoothness issue is given by the mica vs. gold studies, Figs. 7 and 8). Clearly even when the primary mode of friction is the rubbing of opposing boundary layers, and that of its reduction is via boundary lubrication, other modes of energy dissipation during sliding may play a role. The overall friction is clearly the sum of all such modes. These include viscous effects, for example when boundary polymer layers disentangle on sliding; viscoelastic effects when soft substrates are sheared and deformed; and plastic deformation and wear due to asperity contact even when most of the sliding is between the rubbing boundary layers. These additional modes need to be accounted for in any complete picture of the friction [100]. Nonetheless, SFB experiments provide a direct measure of the most basic intrinsic process as the boundary layers - be they liposomes, polymer brushes or trapped hydrated ions - rub past each other. In addition, when soft surfaces-and that includes much of biology-are compressed and made to slide, their softness (which may be quantified as a mechanical modulus < ca. $0.1-1 \mathrm{MPa}$ ) implies that even under moderate compression two such surfaces will be in intimate molecular contact [101]. This is precisely the geometry over the contact area of sliding smooth surfaces within the SFB, and implies that its most basic findings apply to boundary lubrication of soft surfaces.

Finally, we may identify several challenges and opportunities related to hydration lubrication. These include:

(1) What is the precise mechanism by which hydration lubrication operates? Earlier it was assumed qualitatively that it operates via a fluid response to shear of the hydration shells; but do the hydration shells respond in a Newtonian manner to shear (i.e., is the stress $\sigma \sim \dot{\gamma}$, the shear rate)? A more detailed picture, which might be obtained through a wider range of shear rate studies, or spectroscopic approaches, or via computer simulations, is still lacking. The assumption that hydration lubrication will be efficient up to shear rates comparable to the hydration shell relaxation rates also needs to be examined.

(2) What are the ultimate pressures at which this mechanism will apply? The crude estimate provided in this review suggests that pressures up to order 100 MPa might be sustained by suitably hydrated ions. Some insight into this may come from computer simulations, though these hold their own challenges, particularly in water environments. They will depend on the details of the surrounding of the charge (e.g., the microscopic confining surface structure), the way in which the hydration water rearranges under compression, and indeed on the pressure-viscosity coefficient of water in hydration shells, which may differ from that of bulk waterwhere it is approximately zero-and is not, to our knowledge, known.

(3) Can one systematically identify and rank charged groups, and/or their combinations, according to their hydration lubrication efficiency, so that design of efficient boundary lubricants at the molecular level becomes possible? Why, in particular, are phosphocholine groups such excellent hydrationlubrication elements? Could other such groups be predicted?

(4) Would multivalent ions have desirable, possibly tunable properties as boundary lubricants? Multivalent ions have larger hydration energies, and a wide range of relaxation times, but a recent SFB study on mica surfaces immersed in $0.1 \mathrm{M} \mathrm{Ni}^{2+}$ solution suggests that extrapolation of results on monovalent ions to multivalent may not be straightforward [66]: At these salt concentrations the $\mathrm{Ni}^{2+}$ ions were apparently expelled from between the surfaces to be replaced by hydrated protons, so that no hydration lubrication was observed. Other studies [131] indicate that the critical hydration concentration of multivalent ions is in the molar $(1 \mathrm{M})$ range, rather than $\mathrm{mM}$ or less for monovalent alkali metal [57] ions.

(5) The extremely efficient lubrication by phosphocholine-bearing vectors such as brushes or liposomes present both a challenge to our 
understanding and an opportunity for exploitation. It seems clear that, via the hydration lubrication mechanism, such groups must play a central role in biological friction and lubrication processes, although the precise manner in which they do this is not known (and probably differs in different biological environments). Because of their biocompatibility, such groups also have promise, given appropriate delivery vectors, to dramatically reduce friction and wear of biomedical devices or friction between living surfaces in medical treatments. Development of such vectors is in itself a worthy and major challenge.

\section{Acknowledgements}

I thank my students, post-docs, collaborators and colleagues, both past and present, too numerous to list here by name, for their contributions to the work described in this review. I thank the European Research Council (Advanced Grant HydrationLube), the McCutchen Foundation, the Israel Science Foundation, the Ministry of Trade and Industry (Israel) and the Weizmann-UK Research Program (supported by Weizmann Friends in the UK) for their support of this work.

Open Access: This article is distributed under the terms of the Creative Commons Attribution Noncommercial License which permits any noncommercial use, distribution, and reproduction in any medium, provided the original author(s) and source are credited.

\section{References}

[1] Dowson D. History of Tribology. London: Longmans, 1979.

[2] Nosonovsky M. Oil as a lubricant in the Ancient Middle East. Tribology Online 2(2): 44-49 (2007)

[3] Bhushan B. Fundamentals of Tribology and Bridging the Gap Between Macro- and Micro/Nanoscales. Dordrecht: Kluwer Academic, 2001.

[4] Singer I L, Pollock H M. Fundamentals of Friction: Macroscopic and Microscopic Processes. Dordrecht: Kluwer Scientific, 1991.

[5] Tabor D. Friction as a dissipative process. In Fundamentals of Friction: Macroscopic and Microscopic Processes, Pollock I, Ed. Dordrecht: Kluwer, 1992: 3-20.
[6] Tabor D. Friction - The present state of our understanding. J Lubrication Technology 103: 169-179 (1981)

[7] Bowden F P, Tabor D. Friction: An Introduction to Tribology. New York: Anchor Press/Doubleday, 1973.

[8] Bowden F P, Tabor D. The Friction and Lubrication of Solids. Oxford: Oxford University Press, 2001.

[9] Bhushan B. Modern Tribology Handbook, Vols. 1 and 2. Boca Raton: CRC Press, 2001.

[10] Hardy W, Bircumshaw I. Boundary lubrication: Plane surfaces and the limitations of Amontons Law. In Proc. Roy. Soc. , London, UK, 1925: 1-27.

[11] Hardy W B. Collected Works. Cambridge: Cambridge University Press, 1936.

[12] Prandtl L. Ein Gedankenmodell zur kinetischen Theorie der festen Körper. ZAMM 8: 85-106 (1928)

[13] Tomlinson G A. A molecular theory of friction. Phil Mag 7: 905-939 (1929)

[14] Chen Y L, Helm C, Israelachvili J N. Molecular mechanisms associated with adhesion and contact angle hysteresis of monolayer surfaces. J Phys Chem 95: 1073610747 (1991)

[15] Tabor D. The role of surface and intermolecular forces in thin film lubrication. In Microscopic Aspects of Adhesion and Lubrication. Georges J M, Ed. Amsterdam: Elsevier, 1980: 651-679.

[16] Yoshizawa H, Chen Y-L, Israelachvili J. Fundamental mechanisms of interfacial friction. 1. Relation between adhesion and friction. J Phys Chem 97: 4128-4140 (1993)

[17] Bowden F P, Tabor D. The Friction and Lubrication of Solids I. Oxford: Clarendon Press, 1950.

[18] Bowden F P, Tabor D. The Friction and Lubrication of Solids II. Oxford: Clarendon Press, 1964.

[19] Brochard F, de Gennes P G. Shear dependent slippage at a polymer/solid interface. Langmuir 8: 3033-3037 (1992)

[20] Klein J. Shear, friction, and lubrication forces between polymer-bearing surfaces. Annu Rev Mater Sci 26: 581612 (1996)

[21] Klein J, Kumacheva E, Mahalu D, Perahia D, Fetters L. Reduction of frictional forces between solid surfaces bearing polymer brushes. Nature 370: 634-636 (1994)

[22] Schorr P, Kwan T, Kilbey M, Shaqfeh S G, Tirrell M. Shear forces between tethered polymer chains as a function of compression, sliding velocity and solvent quality. Macromolecules 36: 389-398 (2003)

[23] Halperin A, Tirrell M, Lodge T. Tethered chains in polymer microstructures. Advances in Polymer Science $\mathbf{1 0 0}$ 31-71 (1992)

[24] Kampf N, Gohy J F, Jerome R, Klein J. Normal and shear forces between a polyelectrolyte brush and a solid surface. J Polym Sci B - Polym Phys 43: 193-204 (2005) 
[25] Leger L, Raphael E, Hervet H. Surface-anchored polymer chains: Their role in adhesion and friction. Adv Polymer Sci 138: 185-225 (1999)

[26] Wijmans C M, Zhulina E B, Fleer G J. Effect of free polymer on the structure of a polymer brush and interaction between 2 polymer brushes. Macromolecules 27: 32383248 (1994)

[27] Witten T, Leibler L, Pincus P. Stress-relaxation in the lamellar copolymer mesophase. Macromolecules 23: 824829 (1990)

[28] Klein J, Kumacheva E, Perahia D, Mahalu D, Warburg S. Interfacial sliding of polymer-bearing surfaces. Faraday Discuss 98: 173-188 (1994)

[29] Tadmor R, Janik J, Fetters L J, Klein J. Sliding friction with polymer brushes. Phys Rev Lett 91: 115503 (2003)

[30] Raviv U, Tadmor R, Klein J. Shear and frictional Interactions between adsorbed polymer layers is a good solvent. J Phys Chem B 105: 8125-8134 (2001)

[31] Dowson D. Biotribology of natural and replacement synovial joints. In Biomechanics of Diarthroidal Joints. Mow V C, Ratcliffe A, Woo S L-Y, Ed. New York: Springer Verlag, 1990: 305-345.

[32] Dowson D. New joints for the millenium: Wear control in total replacement hip joints. Proceedings of the Institution of Mechanical Engineers, Part H: Journal of Engineering in Medicine 215: 335-358 (2001)

[33] Dowson D, Wright V, Longfield M D. Human joint lubrication. Biomed Eng 4: 160-165 (1969)

[34] McCutchen C W. Lubrication and wear in living and artificial human joints: Physiological lubrication. Proc Inst Mech Eng 181: 55-62 (1967)

[35] McCutchen $\mathrm{C} \mathrm{W}$. The frictional properties of animal joints. Wear 5: 1-17 (1962)

[36] Klein J. Repair or replacement: A joint perspective. Science 323: 47-48 (2009)

[37] Raviv U, Laurat P, Klein J. Fluidity of water confined to sub-nanometre films. Nature 413: 51-54 (2001)

[38] Raviv U, Perkin S, Laurat P, Klein J. Fluidity of water confined down to sub-nanometer films. Langmuir 20: 5322-5332 (2004)

[39] Israelachvili J N, McGuiggan P M. Dynamic properties of molecularly thin liquid films. Science 240: 189-191 (1988)

[40] Klein J, Kumacheva E. Confinement-induced phase transitions in simple liquids. Science 269: 816-819 (1995)

[41] Cotton F A, Wilkinson G. Advanced Inorganic Chemistry. NY: Wiley, 1998.

[42] Briscoe W H, Titmuss S, Tiberg F, Thomas R K, McGillivray D J, Klein J. Boundary lubrication under water. Nature 444: 191-194 (2006)
[43] Chen M, Briscoe W H, Armes S P, Klein J. Lubrication at physiological pressures by polyzwitterionic brushes. Science 323: 1698-1701 (2009)

[44] Raviv U, Giasson S,Kampf N,Gohy J F,Jérôme R,Klein J. Lubrication by charged polymers. Nature 425: 163-165 (2003)

[45] Raviv U, Klein J. Fluidity of bound hydration layers. Science 297: 1540-1553 (2002)

[46] Goldberg R, Schroeder A, Barenholz Y, Klein J. Boundary lubricants with exceptionally low friction coefficients based on 2D close-packed phosphatidylcholine liposomes. Advanced Materials 23: 3517-3521 (2011)

[47] Israelachvili J N. Intermolecular and Surface Forces. Waltham: Academic Press, 2011.

[48] Saluja P P S. Environment of ions in aqueous solutions. Int Rev Sci Electrochemistry, Part 1, Physical Chemistry Series 26: 1-51 (1976)

[49] Derjaguin B V, Churaev N V, Muller V M. Surface Forces. New York: Plenum Publishing Corporation, 1987.

[50] Safran S A. Statistical Thermodynamics of Surfaces, Interfaces and Membranes. New York: Addison-Wesley, 1994.

[51] Silbert G, Ben-Yaakov D, Dror Y, Perkin S, Kampf N, Klein J. Long-ranged attraction between disordered heterogeneous surfaces. Phys Rev Lett 109: 168305 (2012)

[52] Luo J B, Wen S Z, Huang P. Thin film lubrication, Part I: The transtion between EHL and thin film lubrication. Wear 194: 107-115 (1996)

[53] Cui S T, Cummings P T, Cochran H D. Molecular simulation of the transition from liquidlike to solidlike behavior in complex fluids confined to nanoscale gaps. $J$ Chem Phys 114: 7189-7195 (2001)

[54] Jagla E A. Boundary lubrication properties of materials with expansive freezing. Phys Rev Lett 88: 245504 (2002)

[55] Israelachvili J N, Adams G E. Measurement of forces between two mica surfaces in aqueous electrolyte solutions in the range 0-100 nm. $J$ Chem Soc Faraday Trans 179: 975-1001 (1978)

[56] LeNeveu D M, Rand R P, Parsegian V A. Hydration repulsion using osmotic stress technique. Nature 259: 601 (1976)

[57] Pashley R M. Hydration forces between mica surfaces in aqueous electrolyte solutions. $J$ Colloid and Interface Science 80: 153-162 (1981)

[58] Pashley R M. DLVO and hydration forces between mica surfaces in $\mathrm{Li}^{+}, \mathrm{Na}^{+}, \mathrm{K}^{+}$, and $\mathrm{Cs}^{+}$electrolyte solutions: A correlation of double-layer and hydration forces with surface cation exchange properties. J Colloid Interface Sci 83: 531-536 (1981) 
[59] Pashley R M. Hydration forces between mica surfaces in electrolyte solutions. Adv Col Int Sci 16: 57-62 (1982)

[60] Espinosa-Marzal R M, Drobek T, Balmer T, Heuberger M. Hydrated ion ordering in electrical double layers. Phys Chem Chem Phys 14: 6085-6093 (2012)

[61] Leng Y, Cummings P T. Hydration structure of water confined between mica surfaces. $J$ Chem Phys 124: 074711(2006)

[62] Burgess J. Metal Ions in Solution. Chichester: Ellis Horwood Ltd., 1978.

[63] Cowley A C, Fuller N L, Rand R P, Parsegian V A. Measurement of repulsive forces between charged phospholipid bilayers. Biochemistry 17: 3163-3168 (1978)

[64] Donose B C, Vakarelski I U, Higashitani K. Silica surface lubrication by hydrated cations adsorption from electrolyte solutions. Langmuir 21: 1834-1839 (2005)

[65] Perkin S, Chai L, Kampf N, Raviv U, Briscoe W, Dunlop I, Titmuss S, Seo M, Kumacheva E, Klein J. Forces between mica surfaces, prepared in different ways, across aqueous and non-aqueous liquids confined to molecularly thin films. Langmuir 22: 6142-6152 (2006)

[66] Perkin S, Goldberg R, Chai L, Kampf N, Klein J. Dynamics of confined hydration layers. Faraday Discussions 141: 399-413 (2009)

[67] Sakuma H, Otsuki K, Kurihara K. Viscosity and lubricity of aqueous $\mathrm{NaCl}$ solution confined between mica surfaces studied by shear pesonance measurement. Phys Rev Lett 96: 046104 (2006)

[68] Chai L, Goldberg R, Kampf N, Klein J. Selective adsorption of poly(ethylene oxide) onto a charged surface mediated by alkali-metal ions. Langmuir 24: 1570-1576 (2008)

[69] Li J, Zhang C, Luo J. Superlubricity behavior with phosphoric acid-water network induced by rubbing. Langmuir 27: 9413-9417 (2011)

[70] Li J J, Zhang C H, Ma L R, Liu Y H, Luo J B. Superlubricity achieved with mixtures of acids and glycerol. Langmuir 29: 271-275 (2013)

[71] Li J J, Zhang C H, Sun L, Liu Y H, Luo J B. Tribochemistry and superlubricity induced by hydrogen ions. Langmuir 28: 15816-15823 (2012)

[72] Chai L, Klein J. Large area, molecularly smooth $(0.2 \mathrm{~nm}$ rms) gold films for surface forces and other studies. Langmuir 23: 7777-7783 (2007)

[73] Chai L, Klein J. Interactions between molecularly-smooth gold and mica surfaces across aqueous solutions. Langmuir 25: 11533-11540 (2009)

[74] Raviv U, Giasson S, Gohy J-F, Jerome R, Klein J. Normal and frictional forces between surfaces bearing polyelectrolyte brushes. Langmuir 24: 8678-8687 (2008)
[75] Mueller M T, Yan X, Lee S, Perry S S, Spencer N D. Preferential solvation and its effect on the lubrication properties of a surface-bound, brushlike copolymer. Macromolecules 38: 3861-3866 (2005)

[76] Raviv U, Frey J, Sak R, Laurat P, Tadmor R, Klein J. Properties and interactions of physigrafted end-functionalized poly (ethylene glycol) layers. Langmuir 18: 7482-7495 (2002)

[77] Kampf N, Raviv U, Klein J. Normal and shear forces between adsorbed and gelled layers of chitosan, a naturally occurring cationic polyelectrolyte. Macromolecules 37: 1134-1142 (2004)

[78] Harvey N M, Yakubov G E, Stokes J R, Klein J. Normal and shear forces between surfaces bearing porcine gastric mucin, a high-molecular-weight glycoprotein. Biomacromolecules 12: 1041-1050 (2011)

[79] Chen M, Briscoe W H, Armes S P, Cohen H, Klein J. Robust, biomimetic polymer brush layers grown directly from a planar mica surface. ChemPhysChem 8: 1303-1306 (2007)

[80] Chen M, Briscoe W H, Cohen H, Armes S P, Klein J. Polyzwitterionic brushes: extreme lubrication by design. European Polymer Journal 47: 511-523 (2011)

[81] Disalvo E A, Lairion F, Martini F, Almaleck H. Water in biological membranes at interfaces: Does it play a functional role? J Argent Chem Soc 92: 1-22 (2004)

[82] Ishihara K, Nomura H, Mihara T, Kurita K, Iwasaki Y, Nakabayashi N. Why do phospholipid polymers reduce protein adsorption? J Biomed Mater Res 39: 323-330 (1998)

[83] Lis L J, McAlister M, Fuller N, Rand R P, Parsegian V A. Interactions between neutral phospholipid-bilayer membranes. Biophysical J 37: 657-665 (1982)

[84] Nagle J F, Zhang R, Tristram-Nagle S, Sun W, Petrache H I, Suter R M. X-ray structure determination of fully hydrated L alpha phase dipalmitoylphosphatidylcholine bilayers. Biophysical J 70: 1419-1431 (1996)

[85] Pabst G, Rappolt M, Amenitsch H, Laggner P. Structural information from multilamellar liposomes at full hydration: Full q-range fitting with high quality x-ray data. Phys Rev E 62: 4000-4009 (2000)

[86] Yaseen M, Lu J R. The structure of zwitterionic phosphocholine surfactant monolayers. Langmuir 22: 5825-5832 (2006)

[87] Moro T, Moro T, Takatori Y, Ishihara K, Konno T, Takigawa Y, Matsushita T, Chung U I, Nakamura K, Kawaguchi H. Surface grafting of artificial joints with a biocompatible polymer for preventing periprosthetic osteolysis. Nature Materials 3: 829-836 (2004)

[88] Rappolt M, Pabst G, Amenitsch H, Laggner P. 
Salt-induced phase separation in the liquid crystalline phase of phosphatidylcholines. Colloids and Surfaces A-Physicochemical and Engineering Aspects 183-185: 171-181 (2001)

[89] Kobayashi M, Terada M, Takahara A. Polyelectrolyte brushes: A novel stable lubrication system in aqueous conditions. Faraday Discuss 156: 403-412 (2012)

[90] Kobayashi M, Terayama Y, Hosaka N, Kaido M, Suzuki A, Yamada N, Torikai N, Ishihara K, Takahara A. Friction behavior of high-density poly (2-methacryloyloxyethyl phosphorylcholine) brush in aqueous media. Soft Matter 3: 740-746 (2007)

[91] Briscoe W H, Klein J. Friction and adhesion hysteresis between surfactant monolayers in water. The Journal of Adhesion 83: 705-722 (2007)

[92] Busutttil K, Nikogeorgos N, Zhang Z, Geoghegan M, Hunter C A, Leggett G J. The mechanics of nanometrescale molecular contacts. Faraday Discuss. 156: 325-341 (2012)

[93] Briscoe B J, Evans D C B. The shear properties of Langmuir-Blodgett layers. Proc Roy Soc Lond A 380: 389-407 (1982)

[94] Silbert G, Kampf N, Perkin S, Klein J. Time dependence of interactions between a surfactant-coated substrate and a uniformly charged surface. Langmuir 48: 16029-16037 (2012)

[95] Corneci M-C, Dekkiche F, Trunfio-Sfarghiu A-M, Meurisse M-H , Berthier Y, Rieu J-P. Tribological properties of fluid phase phospholipid bilayers. Tribology International 44: 1959-1968 (2011)

[96] Trunfio-Sfarghiu A-M, Berthier Y, Meurisse M-H, Rieu J-P. Role of nanomechanical properties in the tribological performance of phospholipid biomimetic surfaces. Langmuir 24: 8765-8771 (2008)

[97] Immordino M L, Dosio F, Cattel L. Stealth liposomes: Review of the basic science, rationale, and clinical applications. International J of Nanomedicine 1: 297-315 (2006)

[98] Lasic D D, Needham D. The "stealth" liposome: a prototypical biomaterial. Chemical Reviews 95: 2601-2628 (1995)

[99] Goldberg R, Schroeder A, Barenholz Y, Klein J. Interactions between adsorbed hydrogenated soy phosphatidylcholine (HSPC) vesicles at physiologicallyhigh pressures and salt concentrations. Biophysical J. 100: 2403-2411 (2011)

[100] Dowson D. Bio-tribology. Faraday Discuss 156: 9-30 (2012)

[101] Klein J. Molecular mechanisms of synovial joint lubrication. Proc Inst Mech Eng, Part J, Journal of Engineering Tribology 220: 691-710 (2006)
[102] Afoke N Y P, Byers P D, Hutton W C. Contact pressures in the human hip joint. $J$ Bone Joint Surgery 69B: 536-541 (1987)

[103] Hodge W A, Fujant R S, Carlsont K L, Burgesst R G, Harris W H, Manntt R W. Contact pressures in the human hip joint measured in vivo. Proc Natl Acad Sci USA 83: 2879-2883 (1986)

[104] Linn F C. Lubrication of animal joints. II: The mechanism. J Biomech. 1: 193-205 (1968)

[105] Little T, Freeman M, Swanson A. Experiments on friction in the human hip joint. In Lubrication and Wear in Joints. Wright V, Ed. London: Sector Publishing, 1969: 110-116.

[106] Wright V, Dowson D. Lubrication and cartilage. J Anat 121: $107-118$ (1976)

[107] Crocket R, Roos S, Rossbach P, Dora C, Born W, Troxler $\mathrm{H}$. Imaging of the surface of human and bovine articular cartilage with ESEM and AFM. Tribol Lett 19: 311-317 (2005)

[108] Jay G D. Characterization of a bovine synovial fluid lubricating factor: III. The interaction with hyaluronic acid. Conn Tiss Res 28: 245-255 (1992)

[109] Jay G D, Haberstroh K, Cha C-J. Comparison of the boundary-lubricating ability of bovine synovial fluid, lubricin and Healon. J Biomed Mater Res 40: 414-418 (1998)

[110] Jay G D, Harris D A, Cha C-J. Boundary lubrication by lubricin is mediated by O-linked beta(1-3) Gal-GalNAc oligosaccharides. Glcoconjugate J 18: 807-815 (2001)

[111] Swann D, Bloch K J, Swindell D, Shore E. The lubricating activity of human synovial fluids. Arthritis and Rheumatism 27: 552-556 (1984)

[112] Swann D, Silver F H, Slayter H S, Stafford W, E Shore. The molecular structure and lubricating ability of lubricin isolated from bovine and human synovial fluids. Biochem $J$ 225: 195-201 (1985)

[113] Benz M, Chen N, Israelachvili J. Lubrication and wear properties of grafted polyelectrolytes, hyaluronan and hylan, measured in the surface forces apparatus. Journal of Biomedical Materials Research Part A 71A: 6-15 (2004)

[114] Coles J M, Chang D P, Zauscher S. Molecular mechanisms of aqueous boundary lubrication by mucinous glycoproteins. Current Opinion in Colloid and Interface Science 15: 406-416 (2010)

[115] Tadmor R, Chen N H, Israelachvili J N. Thin film rheology and lubricity of hyaluronic acid solutions at a normal physiological concentration. Journal of Biomedical Materials Research 61: 514-523 (2002)

[116] Zappone B, Ruths M, Greene G W, Jay G D, Israelachvili J $\mathrm{N}$. Adsorption, lubrication and wear of lubricin on model surfaces: Polymer brush-like behaviour of a glycoprotein. Biophys J 92: 1693-1708 (2007) 
[117] Han L, Dean D M, Ortiz C, Grodzinsky A J. Lateral nanomechanics of cartilage aggrecan macromolecules. Biophys J 92: 1384-1398 (2007)

[118] Wright V. Lubrication and wear in joints. London: Sector Publishing, 1969.

[119] Maroudas A. Hyaluronic acid films. In: lubrication and wear in living and artificial human joints. Proc Inst Mech Eng 181:122-124 (1967)

[120] Rutjes A W, Jüni P, da Costa B R, Trelle S, Nüesch E, Reichenbach S. Viscosupplementation for osteoarthritis of the knee: A systematic review and meta-analysis. Ann Intern Med 157: 180-191 (2012)

[121] Seror J, Merkher Y, Kampf N, Collinson L, Day A J, Maroudas A, Klein J. Articular cartilage proteoglycans as boundary lubricants: Structure and frictional interaction of surface-attached hyaluronan and hyaluronan-aggrecan complexes. Biomacromolecules 12: 3432-3443 (2011)

[122] Seror J, Merkher Y, Kampf N, Collinson L, Day A J, Maroudas A, Klein J. Normal and shear interactions between hyaluronan-aggrecan complexes mimicking possible boundary lubricants in articular cartilage in synovial joints. Biomacromolecules 13: 3823-3832 (2012)

[123] Hills B A. Boundary lubrication in vivo. Proc Inst Mech Eng Part H - J Engineering in Medicine 214: 83-94 (2000)

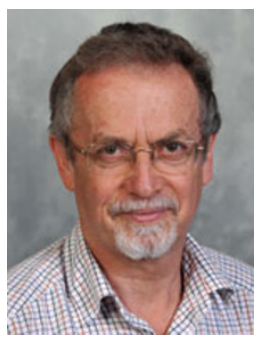

Jacob Klein, born 1949, is the Herman Mark Professor of Polymer Physics at the Weizmann Institute in Israel.

Klein gained his BA in Physics at the University of Cambridge, where in 1977 he also received his M.A. and PhD at the Cavendish Laboratory. He did his postdoc at the Weizmann Institute in Israel, and from 1980-1984 was a Senior Scientist at the Weizmann Institute and a University Demonstrator at the Cavendish Laboratory. In 1984 he was appointed Professor at the Weizmann Institute (full Professor from 1987), and subsequently headed its Polymer Research department and was Chairman of its Scientific Council. From 2000-2007 Klein was the
[124] Hills B A. Surface active phospholipid: A Pandora's box of clinical applications. II: Barrier and lubricating properties. Internal Medicine J 32: 242-251 (2002)

[125] Hills B A, Butler B D. Surfacants identified in synovial fluid and their ability to act as boundary lubricants. Annals of the Rheumatic Diseases 43: 641-648 (1984)

[126] Hills B A, Jay G D. Identity of the joint lubricant. $J$ Rheumatology 29: 200-201 (2002)

[127] Williams III P F, Powell G L, LaBerge M. Sliding friction analysis of phosphatidylcholine as a boundary lubricant for articular cartilage. Proc Inst Mech Eng Part H-J Engineering in Medicine 207: 59-66 (1993)

[128] Bartlett P N. General discussion. Faraday Discuss 156: 203-222 (2012)

[129] Goldberg R, Chai L, Perkin S, Kampf N, Klein J. Breakdown of hydration repulsion between charged surfaces in aqueous Cst solutions. Phys Chem Chem Phys 10: 4939-4945 (2008)

[130] Goldberg R, Klein J. Liposomes as lubricants: Beyond drug delivery. Chemistry and Physics of Lipids 165: 374-381 (2012)

[131] Pashley R M, Israelachvili J N. DLVO and hydration forces between mica surfaces in $\mathrm{Mg}^{2+}, \mathrm{Ca}^{2+}, \mathrm{Sr}^{2+}$ and $\mathrm{Ba}^{2+}$ chloride solutions. J Colloid Interface Sci 97: 446-455 (1984)

Dr. Lee's Professor of Chemistry at the University of Oxford and Head of its Physical and Theoretical Chemistry Laboratory (2000-2005). His interests in soft matter have ranged from the dynamics and interfacial properties of polymers to the behaviour of confined fluids and biological lubrication, and, more recently, tissue engineering. Klein has published over 200 papers, including over 20 in Science and Nature. His honours include the Charles Vernon Boys Prize of the Institute of Physics, UK (1984), the High Polymer Physics Prize of the American Physical Society (1995), the 2010 Prize of the Israel Chemical Society and the 2011 Soft Matter and Biophysical Chemistry Award of the UK Royal Society of Chemistry and the 2012 Tribology Gold Medal. In 2009, he received an ERC Advanced Grant. 